\title{
Technology Delivered Interventions for Depression and Anxiety in Children and Adolescents: A Systematic Review and Meta-analysis
}

\author{
Rebecca Grist $^{1,3} \cdot$ Abigail Croker $^{2} \cdot$ Megan Denne $^{2} \cdot$ Paul Stallard $^{1,2}$
}

Published online: 18 September 2018

(c) The Author(s) 2018

\begin{abstract}
Depression and anxiety are common during adolescence. Whilst effective interventions are available treatment services are limited resulting in many adolescents being unable to access effective help. Delivering mental health interventions via technology, such as computers or the internet, offers one potential way to increase access to psychological treatment. The aim of this systematic review and meta-analysis was to update previous work and investigate the current evidence for the effect of technology delivered interventions for children and adolescents (aged up to 18 years) with depression and anxiety. A systematic search of eight electronic databases identified 34 randomized controlled trials involving 3113 children and young people aged 6-18. The trials evaluated computerized and internet cognitive behavior therapy programs (CBT: $n=17$ ), computer-delivered attention bias modification programs (ABM: $n=8)$ cognitive bias modification programs (CBM: $n=3)$ and other interventions $(n=6)$. Our results demonstrated a small effect in favor of technology delivered interventions compared to a waiting list control group: $g=0.45$ [95\% CI 0.29, 0.60] $p<0.001$. CBT interventions yielded a medium effect size $(n=17, g=0.66$ [95\% CI $0.42-0.90] p<0.001)$. ABM interventions yielded a small effect size $(n=8, g=0.41[95 \% \mathrm{CI}$ $0.08-0.73] p<0.01)$. CBM and 'other' interventions failed to demonstrate a significant benefit over control groups. Type of control condition, problem severity, therapeutic support, parental support, and continuation of other ongoing treatment significantly influenced effect sizes. Our findings suggest there is a benefit in using CBT based technology delivered interventions where access to traditional psychotherapies is limited or delayed.
\end{abstract}

Keywords Depression $\cdot$ Anxiety $\cdot$ Child $\cdot$ Adolescent $\cdot$ Technology $\cdot$ Review

\section{Introduction}

Anxiety and depression in children and young people are common (Merikangas et al. 2009). Over a six-month period up to $8 \%$ of adolescents suffer from a major depressive disorder and cumulatively, by the age of 18 , up to $20 \%$ will experience at least one clinically significant depressive episode

Work was conducted at the Department for Health, 6.19 Wessex House, University of Bath, BA2 7AY, UK

Rebecca Grist

r.grist@brighton.ac.uk

1 Department for Health, University of Bath, 6.19 Wessex House, Bath BA2 7AY, UK

2 Oxford Health NHS Foundation Trust, Child, and Adolescent Mental Health Service, Temple House, Keynsham, UK

3 School of Applied Social Science, University of Brighton, Mayfield House, Falmer, Brighton BN1 9PH, UK
(Costello et al. 2006; Merry et al. 2012a). Recurrence is common with up to $75 \%$ experiencing a subsequent episode of depression within 5 years (Lewinsohn et al. 2000). Adolescent depression has a negative impact on relationships, developmental trajectories, schooling, and educational attainment, and increases the risk of attempted and completed suicide (Birmaher et al. 1996; Fletcher 2008; Gould et al. 2003). Similarly, up to $10 \%$ of children and $20 \%$ of adolescents will suffer from an anxiety disorder (Essau et al. 2012). Anxiety disorders are associated with poor academic performance and adversely affect relationships, along with increasing the risk of depression, illicit drug dependence and educational under-achievement in young adulthood (KimCohen et al. 2003; Woodward and Fergusson 2001).

Psychological therapies are effective in the treatment of anxiety and depressive disorders in children and adolescents (James et al. 2013; Reynolds et al. 2012). However, many children and young people with mental health problems do not receive specialist treatments (Merikangas et al. 2011; 
Ford et al. 2005). Despite initiatives to increase the capacity of child mental health services to deliver evidence-based interventions demand for face to face therapy continues to outstrip capacity (Fonagy et al. 2017). This has led to interest in the use of information and communications technology (e-mental health) to increase capacity to support and improve the mental health of children and young people (Riper et al. 2010; Boydell et al. 2014; Ebert et al. 2015; Hollis et al. 2017).

E-mental health embraces a range of digital technologies that deliver interventions via computers, or through webbased platforms via mobile tablets or smartphones (Hollis et al. 2017). Advantages of digital technologies include greater reach to geographically isolated populations; flexible access; increased convenience; fewer visits to specialist clinics; greater privacy and anonymity; enhanced treatment fidelity; rapid scalability; and low-cost delivery (Clarke et al. 2015; MacDonell and Prinz 2017). There are also several concerns about the use of digital technologies including the absence of a strong motivating and supportive therapeutic relationship; negative professional attitudes to their use; technology failure; questions about their effectiveness in treating severe mental health problems; high rates of attrition; and concerns about data security and quality control (Lal and Adair 2014).

It has been suggested that digital technology may be particularly appealing to adolescents who are typically early adopters and regular users of new technologies (Johnson et al. 2015). In the UK, $83 \%$ of 12-15-year olds have their own smartphone, 55\% have their own tablet, with 99\% going online for almost $21 \mathrm{~h}$ per week (Ofcom 2013). In the US, 93\% of 12-17-year olds have access to a desktop or laptop computer with $74 \%$ having internet access (Madden et al. 2013). Whilst the development of technology to support mental health interventions with children is still in its infancy, results from studies with adults have shown that internet and computer-delivered interventions can be effective for the treatment of depression and anxiety (Andersson and Cujipers 2009; Andrews et al. 2010; Griffiths et al. 2010).

Although e-mental health interventions are fast-developing, those specifically developed for children with anxiety and depression are more limited (Richardson et al. 2010; Calear and Christensen 2010; Pennant et al. 2015). Small RCTs have demonstrated that established evidence-based face to face CBT anxiety interventions such as Cool Teens can be effective when delivered via a CD-ROM with minimal therapist support (Wuthrich et al. 2012). Similarly, online CBT anxiety programs such as BRAVE were found to be very acceptable to young people and as effective as face to face CBT (Spence et al. 2011). In terms of depression, encouraging results have been reported for Stressbusters, a computerized CBT program (Smith et al. 2015; Wright et al. 2017) and a computer game (SPARX) used both as an intervention and as a prevention program (Merry et al. 2012a, b; Perry et al. 2017).

Novel interventions including attentional bias modification training (ABM) and cognitive bias modification (CBM) which attempt to reduce the attentional and cognitive biases associated with depression and anxiety have also been investigated (Bar-Haim et al. 2011; LeMoult et al. 2017). In a review, Pennant et al. (2015) note that although ABM and CBM improved the outcomes of attention and interpretation bias, no conclusive benefits were found for depression and anxiety outcomes. Further investigation into these interventions is therefore warranted.

Systematic reviews have explored the efficacy of digital technologies in the treatment of depression and anxiety disorders in children and adolescents. The first reviews published in 2010 identified only a handful of very small studies resulting in the authors being unable to draw any conclusions other than noting that this appears to be a promising area to explore (Calear and Christensen 2010; Richardson et al. 2010). More recent reviews identified more studies but included young adults (up to the age of 25) and included both prevention and treatment studies (Hollis et al. 2017; Pennant et al. 2015). Although the authors were cautiously positive they noted that the evidence was inconclusive, and that the research suffered from several methodological limitations. The review by Pennant et al. (2015) was updated by Hollis et al. (2017) and included studies up until 1/11/2015. However, given the small number of anxiety and depression treatment studies identified during the update period, the authors did not undertake any specific analysis of these problem groups. Given that E-health is a rapidly developing area we do not have any contemporary systematic analysis of the effects of technological interventions for children and young people (up to age 18) with depression or anxiety.

This review will address this issue and will conduct a meta-analysis exploring the effect of technological interventions for children and young people up to the age of 18 with anxiety and depression. We will undertake sub-group analyses to investigate the effects of anxiety and depression, control condition, problem severity, theoretical basis, therapist assistance, and parental involvement, and whether other interventions were also provided.

\section{Method}

\section{Study Identification}

Eight electronic databases were systematically searched for publications between January 2013 and September 2017. These included: APA PsychNET, Embase, Google Scholar, PubMed, Science Direct, Scopus, Social Policy and Practice 
and Web of Science. Databases of main journals JMIR, Cyberpsychology, Behavior and Social Networking and Internet Interventions were also searched using key search terms. A systematic search of each database was conducted using a combination of search terms relating to the mental health problems targeted, the medium of intervention delivery (computerized, internet, smartphone), population age (child, teenage, adolescent), and the type of study. Databasespecific filters such as human population, English language, and age groups were applied where available. Appendix A contains the full list of search strings by databases. Reference lists of included articles were also screened for potentially relevant studies. One researcher (R.G.) conducted the systematic identification, screening, and checking for eligibility of full-text articles for inclusion. This process was then independently conducted by two researchers (A.C/ and M.D.) with disagreements discussed and a consensus on inclusion or exclusion reached.

\section{Inclusion Criteria}

We included randomized controlled trials of technology delivered psychological interventions for use by children and adolescents for depression or anxiety disorders. Studies were included if the sample was 18 years old or under. Studies with over 18's were included if the mean age of the sample was 18 or less. We included studies if the sample were assessed to have a diagnosed anxiety or depression disorder or had elevated symptoms which were of mild to moderate severity. This must have been diagnosed by a clinician or assessed by the research team by diagnostic interview or screening for cut off scores on an anxiety or depression questionnaire. The intervention needed to be primarily delivered via technology such as computers, CD-ROM, the internet, smartphones, or virtual reality. Technologies which only augmented traditional face to face therapies or did not constitute a significant proportion of the delivery were excluded. Only randomized controlled trials (RCT) were included. As per previous work (e.g., Pennant et al. 2015), there were no restrictions placed on the theoretical basis of the intervention. We included RCT's which compared an intervention to gold standard face to face CBT, other therapeutically 'active' conditions, attention/placebo training conditions and waitlist controls.

\section{Exclusion Criteria}

Studies were excluded if they did not meet the inclusion criteria described. Studies of universal preventative interventions and studies in which the mental health of the sample was not screened were excluded. Articles were also excluded if the intervention was primarily aimed at parents of children with depression or anxiety and did not involve a component aimed at the children. Interventions in which therapists provided 'live' therapy over the internet either via video conferencing or instant messaging were excluded.

\section{Data Extraction}

Study information including study characteristics, participant information, and mental health outcomes was extracted and included in an Excel spreadsheet. Study characteristics included authors, year of publication, the country in which the study was conducted, and sample sizes. Study characteristics also included how participants were recruited and whether participants were permitted to continue ongoing pharmacological or psychological interventions. Participant information included sample age, primary mental health problem and whether this was screened as elevated or fully diagnosed. To be consistent with the age classification adopted by Pennant et al. (2015) which reflects the transition in the UK from primary to secondary school, studies were classified as focusing solely on children (aged 5-11 years), adolescents (12-18 years), or a mixed age group. Program details included the program name, theoretical basis, where the program was delivered and whether there was any parent support.

Information on therapist support was also extracted. This involved classifying each program according to therapist support as outlined by Newman et al. (2011). Programs could either be: (1) self-administered (SA-therapist contact for assessment at most); (2) predominantly self-administered (PSH-giving initial therapeutic rationale, direction on how to use the program and periodic check-ins, $<90 \mathrm{~min}$ of time); and(3) minimal contact therapy (MCT-active involvement of therapist, help in applying specific therapeutic techniques, $>90$ min of time). Studies were categorized according to therapist support only. Therefore, interventions that provided technical assistance, but not therapeutic support were categorized as self-administered.

For the meta-analysis, the mental health outcomes were the means $(M)$ and standard deviations $(S D)$ on the primary outcome measure of anxiety and/or depression symptoms at post-intervention. When trials used a wait-list control condition (WLC) and an 'active' control condition (such as individual or group CBT) outcomes from both conditions were extracted. Outcomes from the WLC were used as the comparator in the main meta-analysis and sub-group analyses. A specific sub-group analysis was undertaken to compare technological delivered interventions against different categories of control condition. Information on program completion was extracted by obtaining the number of participants who were allocated to and completed the intervention condition within the allotted time frame. 


\section{Quality Assessment}

The quality of each study was assessed according to the Cochrane Collaboration's Risk of Bias Tool. This was conducted by one researcher and then checked by two researchers independently. Disagreements were discussed and resolved to meet a consensus. Studies were assigned either 'low risk', 'unclear risk', or 'high risk' status regarding several domains. These included: selection bias, performance bias, detection bias, attrition bias, reporting bias and final 'other' category of identifiable biases.

\section{Data Analysis}

The statistical software package Review Manager Version 5.3 (Review Manager; The Cochrane Collaboration 2014) was used to conduct the meta-analysis. Post-intervention means standard deviations and sample sizes were entered for the intervention and control conditions of each study. A random-effects meta-analysis was conducted using the standardized mean differences (SMD) to calculate effect size (Hedges' $g$ ). To calculate the heterogeneity of effect sizes the Q statistic and $I^{2}$ statistic was used. A significant Q statistic implies significant heterogeneity indicating more variation in effect sizes that can be attributed to chance alone. The $I^{2}$ statistic expresses the heterogeneity as a percentage, with values of $25 \%$ associated with low heterogeneity, $50 \%$ moderate and $75 \%$ high heterogeneity (Crombie and Davies 2009).

Sub-group analyses were conducted to investigate the influence of (1) control condition (face to face CBT, other therapeutically active control, attention/placebo control and wait-list), (2) mental health problem (anxiety or depression), (3) problem severity (confirmed diagnosis or elevated symptoms), (4) theoretical basis of intervention (CBT, ABM, CBM, other), (5) therapist support (SH, PSH, MCT), (6) active parental involvement (yes or no) and (7) continuation of other treatment for depression or anxiety (yes or no).

\section{Results}

\section{Study Selection}

The systematic literature search yielded 2167 results, of which 2092 were excluded based on screening the abstract, title and duplicate removal. A total of 75 full-text articles were assessed for inclusion; 41 were excluded leaving 34 studies for inclusion in the meta-analysis. Figure 1 details the results at each stage and reasons for exclusion.

\section{Study Characteristics}

Tables 1 and 2 contain the main study characteristics and references of studies included in the meta-analysis contains full references of all included studies. Altogether, the 34 studies included 3113 children and adolescents $(n=1517$ in intervention conditions and $n=1596$ in control conditions) with sample sizes ranging between 19 and 257. Participants were aged between 6 and 18 years old. Some studies $(n=5)$ included participants over 18 (maximum age 22) however for each study the mean age of the sample was under 18 . For the other studies, three involved a study sample of children (5-12 years), 18 involved an adolescent population (13-18 years) with the remaining 13 studies involving a mix of children and adolescents (5-18 years). Studies were conducted in the Netherlands $(n=8)$, Australia $(n=8)$, China $(n=3)$, Sweden $(n=3)$, the UK $(n=3)$, the USA $(n=2)$, Israel $(n=2)$, New Zealand $(n=2)$, Canada $(n=1)$, Ireland $(n=1)$, and Thailand $(n=1)$.

In terms of comparison groups (see Table 2) four studies compared the intervention to a face to face CBT group (school-based group CBT, individual CBT and group-based CBT; Poppelaars et al. 2016; Schoneveld et al. 2017; Spence et al. 2011; Sportel et al. 2013). All but the Schoneveld et al. (2017) study also included a wait-list control group. Three studies utilized control groups classified as a 'other therapeutic control'. These conditions included non-CBT therapeutic content or processes e.g., a single session computerized supportive therapy (Schleider and Wesiz 2017), EMDR (Muris et al. 1998) and treatment as usual, the majority of which was counseling (Merry et al. 2012a, b). Fourteen studies included an attention or placebo comparison group comprised of placebo or neutral attention training $(n=8$; De Voogd et al. 2017 also included a wait-list control), an anti- smoking website $(n=1)$, computerized psychoeducation program $(n=1)$, video games $(n=2)$, usual recreational activities $(n=1)$, and self-help websites with no CBT content $(n=1)$. Finally, 17 studies compared technology delivered interventions to wait-list control conditions.

Continuation of psychological or pharmacological treatment for depression or anxiety was permitted in seven trials $(n=6)$. Several $(n=16)$ trials explicitly forbade either ongoing psychological or pharmacological treatment or both, during the study. For the remainder of the studies ongoing psychological and pharmacological treatment was not reported $(n=12)$.

\section{Mental Health Problem Characteristics}

The majority of interventions $(n=20)$ targeted anxiety disorders (social anxiety disorder-specific $n=5$, OCD specific $n=1$, spider phobia $n=1$, range of anxiety disorders $n=13$ ). Ten interventions targeted depression with four being 
Fig. 1 PRISMA flow diagram of results and publication selection

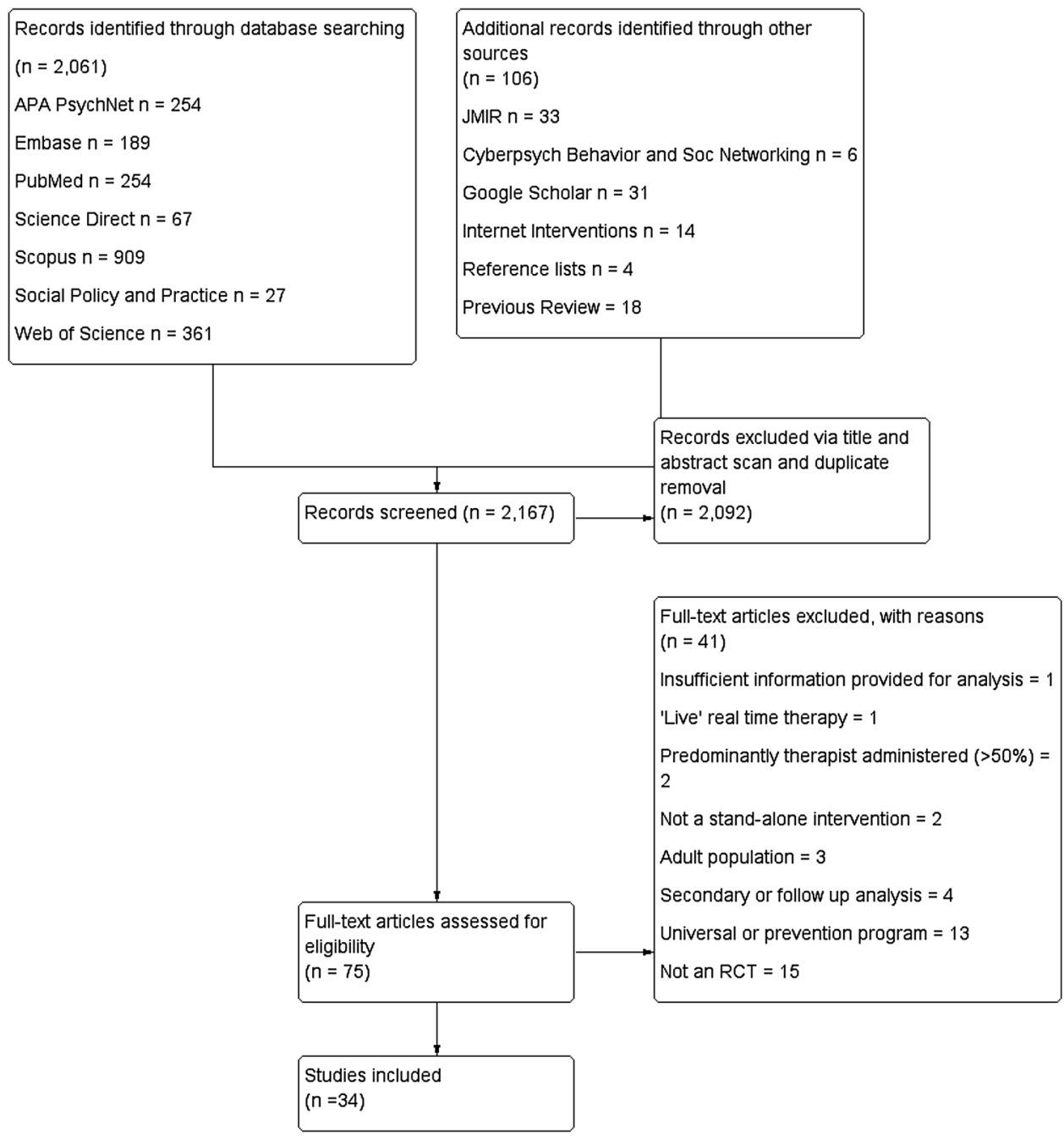

transdiagnostic interventions targeting both anxiety and depression. Over half of the interventions $(n=18)$ involved participants with a confirmed diagnosis of depression or anxiety with the remainder $(n=16)$ including participants with elevated symptoms of depression or anxiety.

\section{Intervention Characteristics}

Interventions were computerized and internet-based CBT $(n=17)$, attention bias modification training $(n=8)$, cognitive bias modification training $(n=3)$, and other interventions $(n=6)$. The other interventions included an internet-based acceptance and commitment therapy program, problem-solving therapy, video games utilizing neuro-feedback, bio-feedback, and emotion regulation training. Less than half of the programs involved guidance and contact from a therapist ( $n=9 \mathrm{MCT}$ and $n=2 \mathrm{PSH}$ ) with most programs being self-administered with no therapist support $(n=23)$. Some programs incorporated some form of parent support $(n=9)$, but the majority did not require any active parental involvement $(n=23)$. For the remainder of the studies, this information was unclear $(n=2)$.

\section{Computerized and Internet-Based CBT}

Several $(n=17)$ studies investigated the use of 13 computerized and internet-based CBT programs for depression and anxiety with five focusing on participants with elevated symptoms of depression: Grasping the Opportunity (CATCH-IT; Ip et al. 2016); SPARX (Merry et al. 2012a, b; Poppelaars et al. 2016); Stressbusters (Smith et al. 2015; Wright et al. 2017), The Journey (Stasiak et al. 2014) and one unnamed guided CCBT program (Wannachaiyakul et al. 2017). Participants with diagnosed anxiety disorders were involved in $(n=8)$ programs: BRAVEONLINE (Conaughton et al. 2017; March et al. 2009; Spence et al. 2011); BiP-OCD (Lenhard et al. 2017); Cool Teens (Wuthrich et al. 2012); Think Feel Do (Stallard et al. 2011) and four unnamed programs, two for social anxiety 


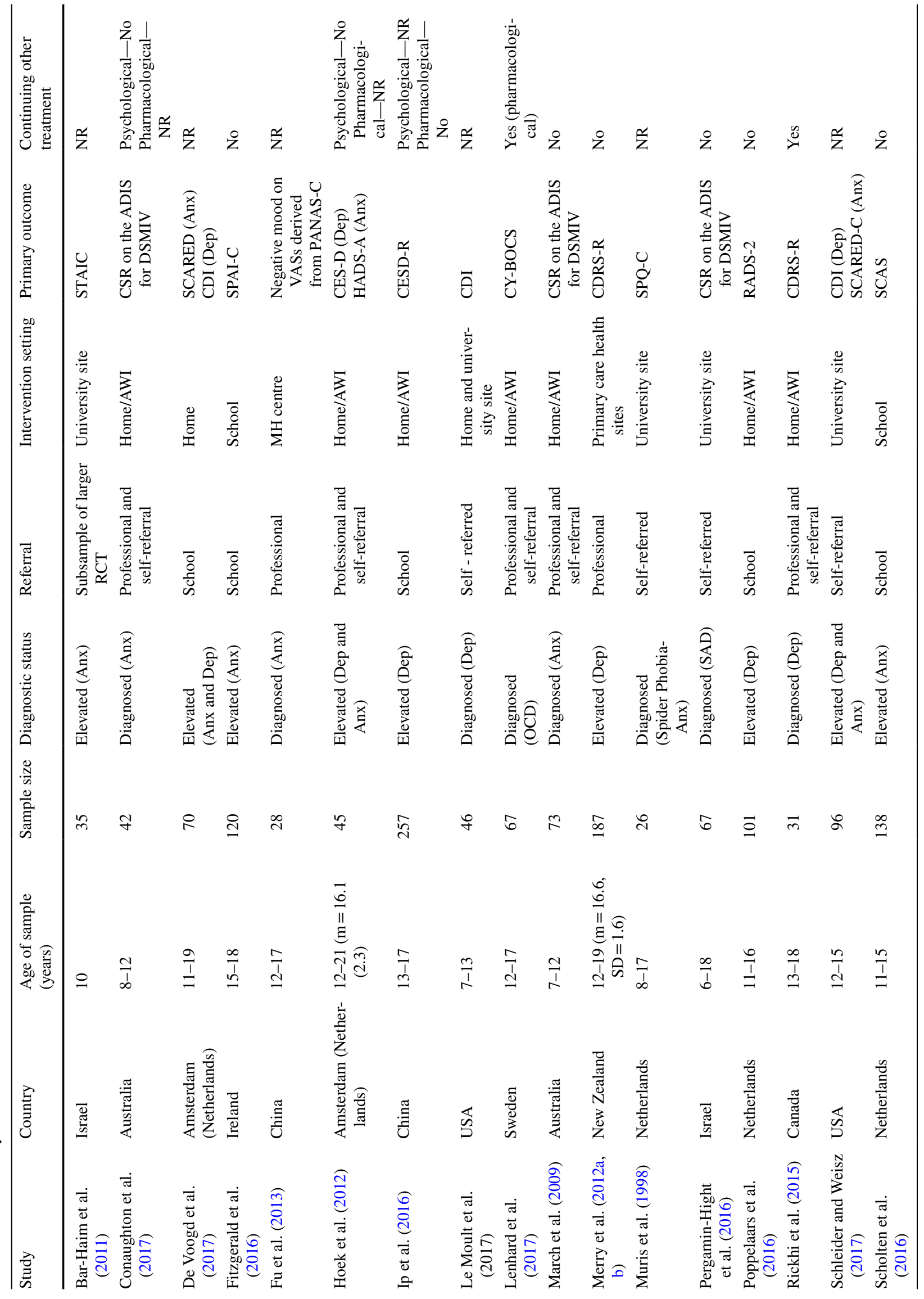




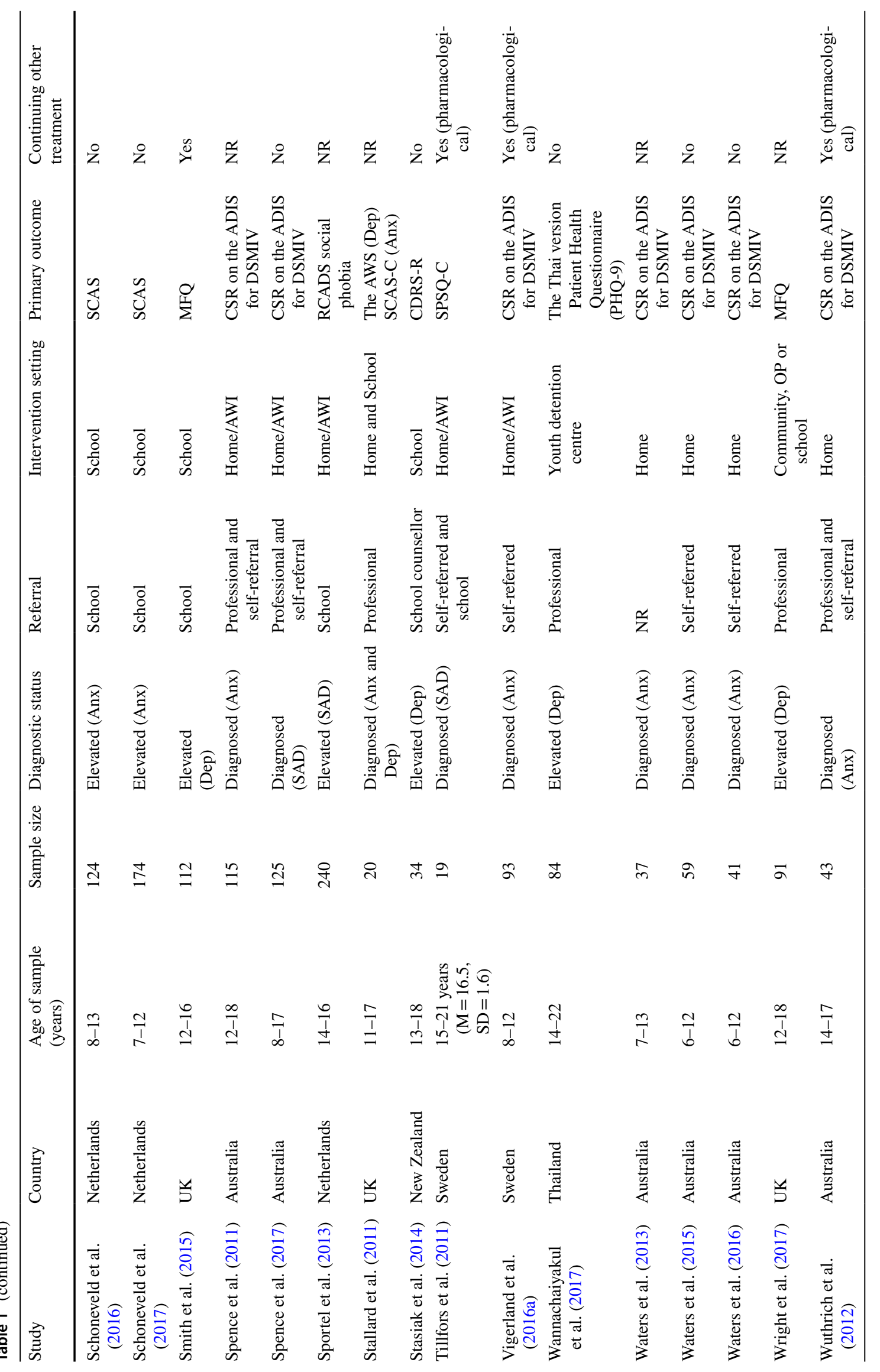




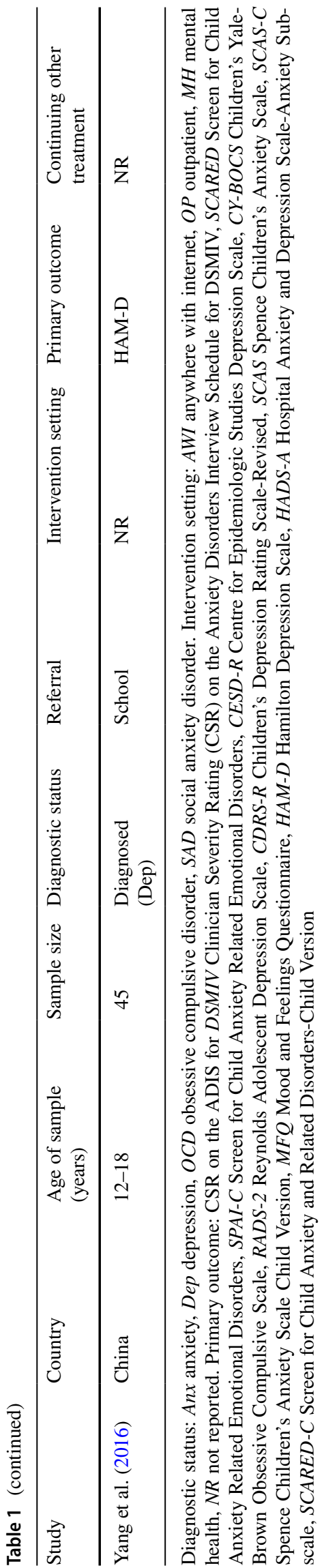

disorder (Spence et al. 2017; Tillfors et al. 2011), one for spider phobia (Muris et al. 1998) and one for children with a range of anxiety disorders (Vigerland et al. 2016a) The majority of programs were therapist-assisted $(n=10)$ with seven including parents.

\section{Attention Bias Modification Training (ABMT)}

The use of attention bias modification training was evaluated in eight trials. One included individuals with a diagnosis of depression, four included individuals diagnosed with anxiety, and three with elevated symptoms of anxiety. Length of ABM training varied from four sessions (Bar-Haim et al. 2011; Fitzgerald et al. 2016), eight sessions (De Voogd et al. 2017; Pergamin-Hight et al. 2016) to 12 sessions (Waters et al. 2013, 2015, 2016; Yang et al. 2016). All the ABMT programs were unguided and did not provide any ongoing clinical support from a therapist. The Waters et al. 2015 and 2016 trials were the only ABMT studies to involve active parental input and were the only ABMT trials to use wait-list controls as a comparison group. All other trials compared ABMT to a placebo training.

\section{Other Technology-Based Interventions}

The remaining nine studies evaluated eight separate programs. Of these, three investigated computer and internetdelivered cognitive bias modification interventions, one for depression (LeMoult et al. 2017), one for anxiety disorders (Fu et al. 2013), and one for social anxiety (Sportel et al. 2013). None of the interventions were therapist or parent assisted.

A trial investigated a 'spiritually informed' 8-week internet intervention called The LEAP project (Rickhi et al. 2015). The LEAP program aimed to treat depression by using spiritually informed principles such as forgiveness, gratitude, and compassion. It did not involve any therapist or parent support and was compared to a wait-list control. Adolescents in this trial had a diagnosis of depression and were self-referred. They were permitted to continue ongoing psychological or pharmacological interventions for depression.

One trial investigated a single session 30-min, computerguided growth mindset intervention (Schleider and Weisz 2017) and another an internet-based (guided) problem-solving therapy intervention (Hoek et al. 2012).

Finally, three trials evaluated two video games used to treat anxiety (Dojo, Scholten et al. 2016 and Mindlight; Schoneveld et al. 2016, 2017). Dojo is a 3D immersive video game specifically designed for reducing anxiety in adolescents and incorporates emotion regulation training and heart rate variability (HRV) biofeedback. Dutch schoolchildren screened to have elevated anxiety symptoms played Dojo or a control game (Rayman) six times over three weeks in a 


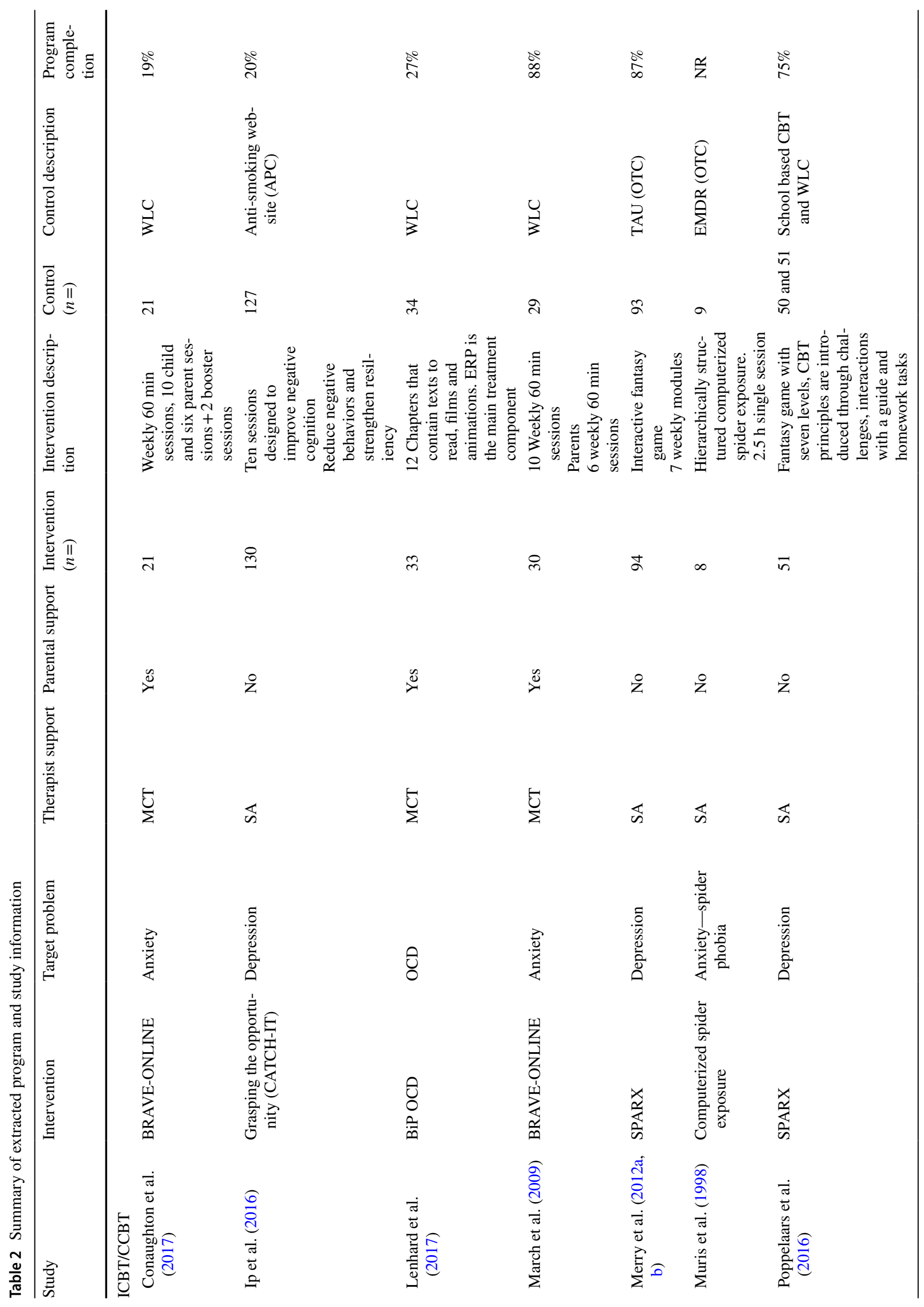




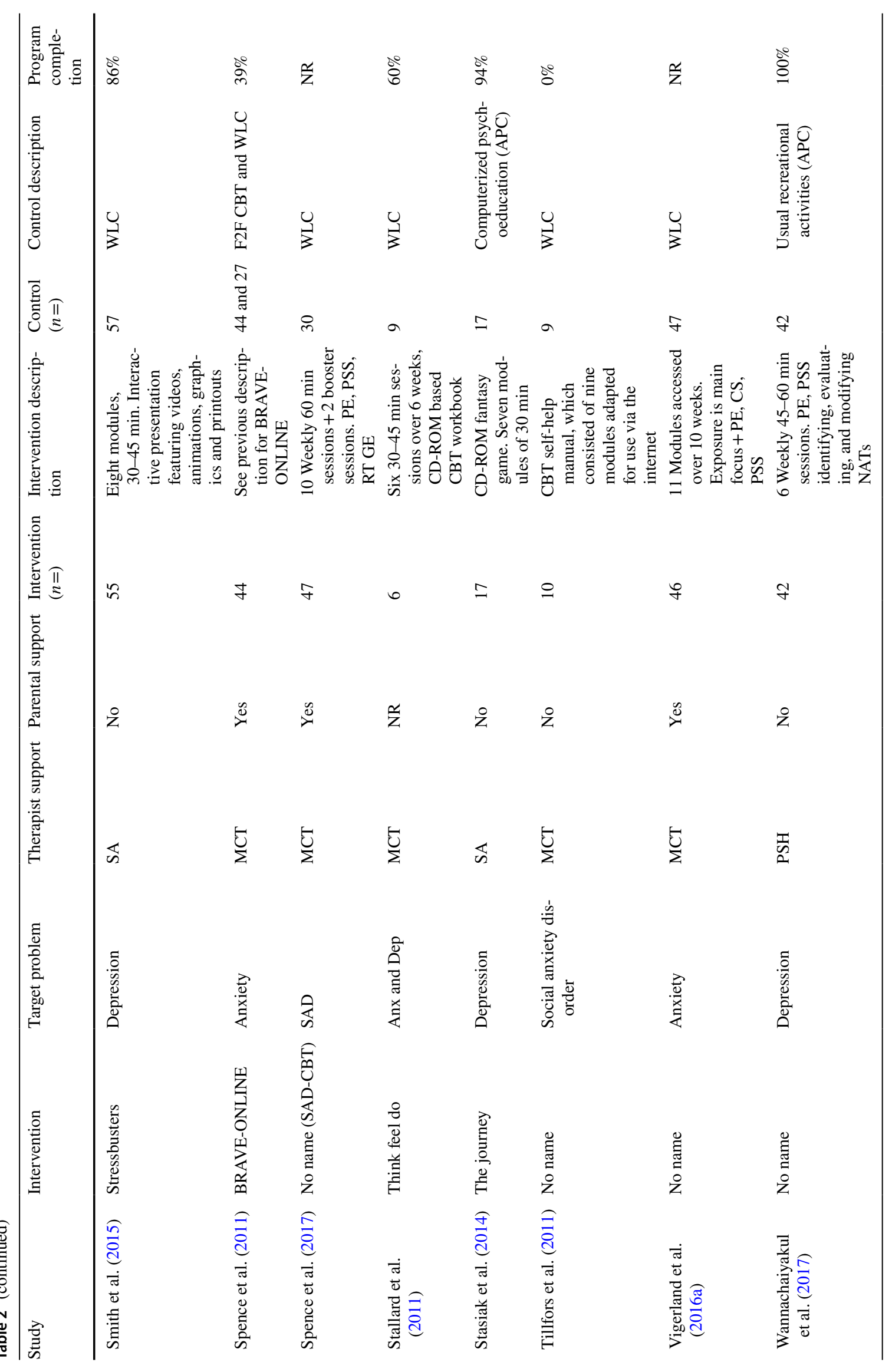


Clinical Child and Family Psychology Review (2019) 22:147-171

157

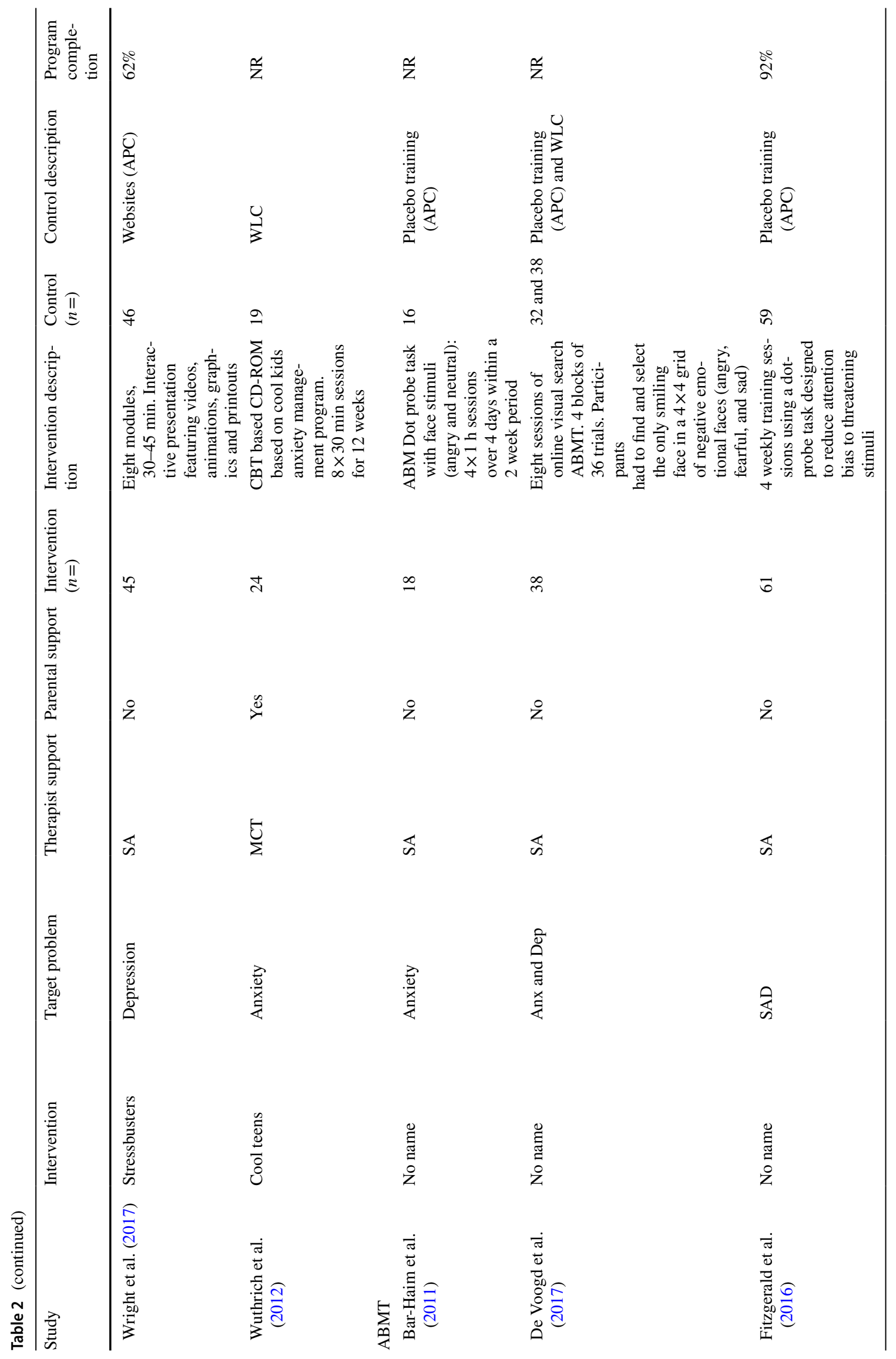

Springer 


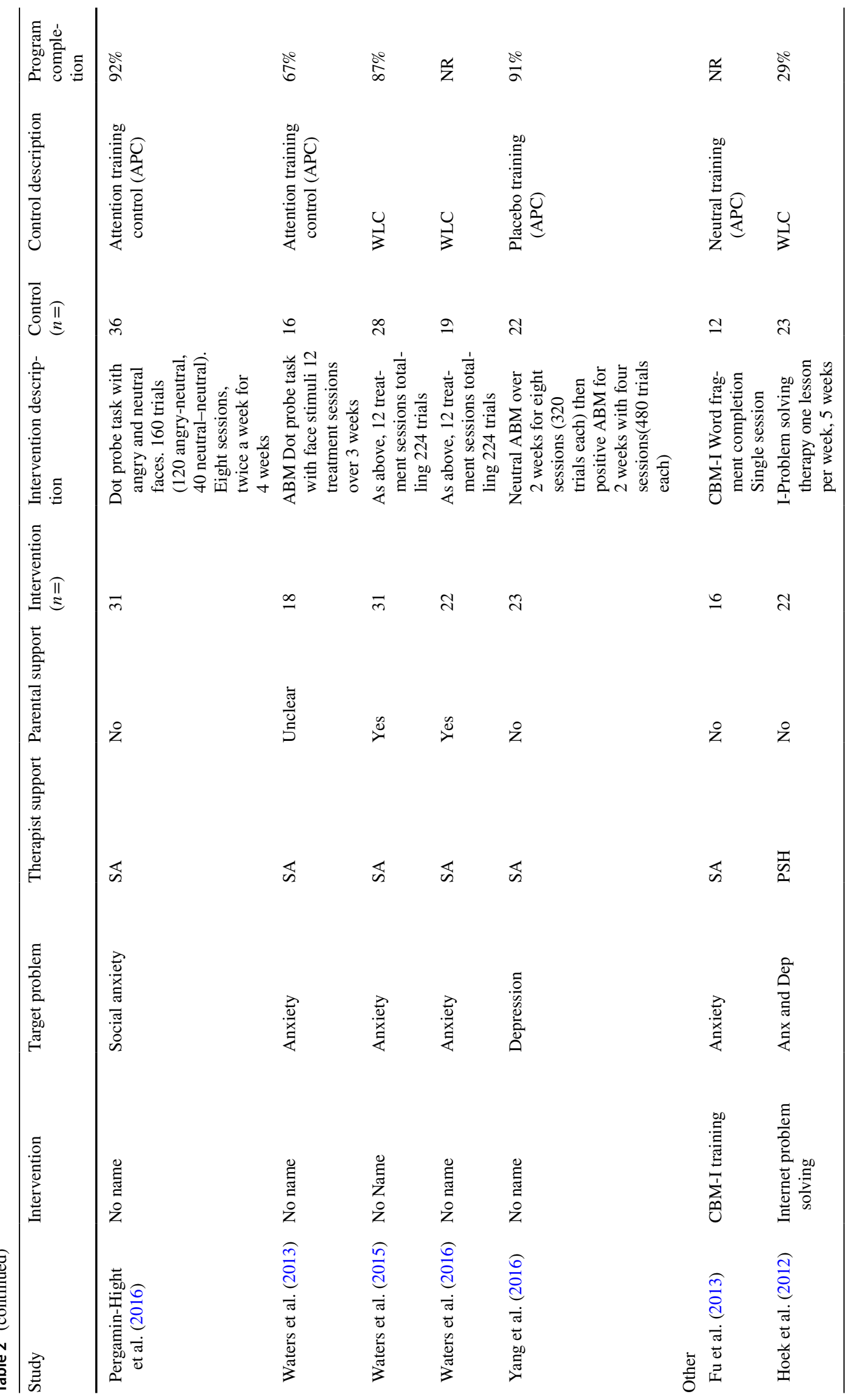




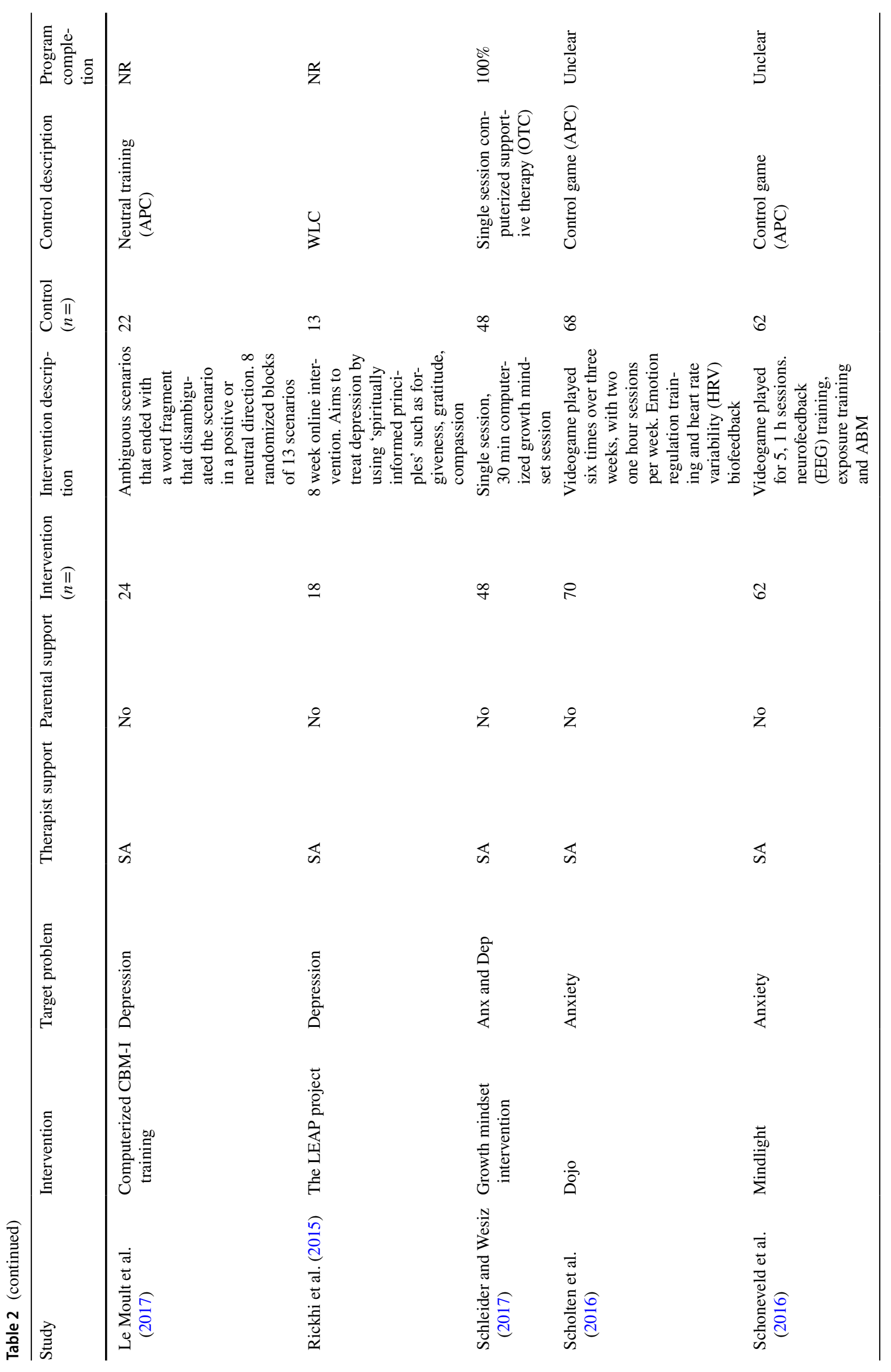




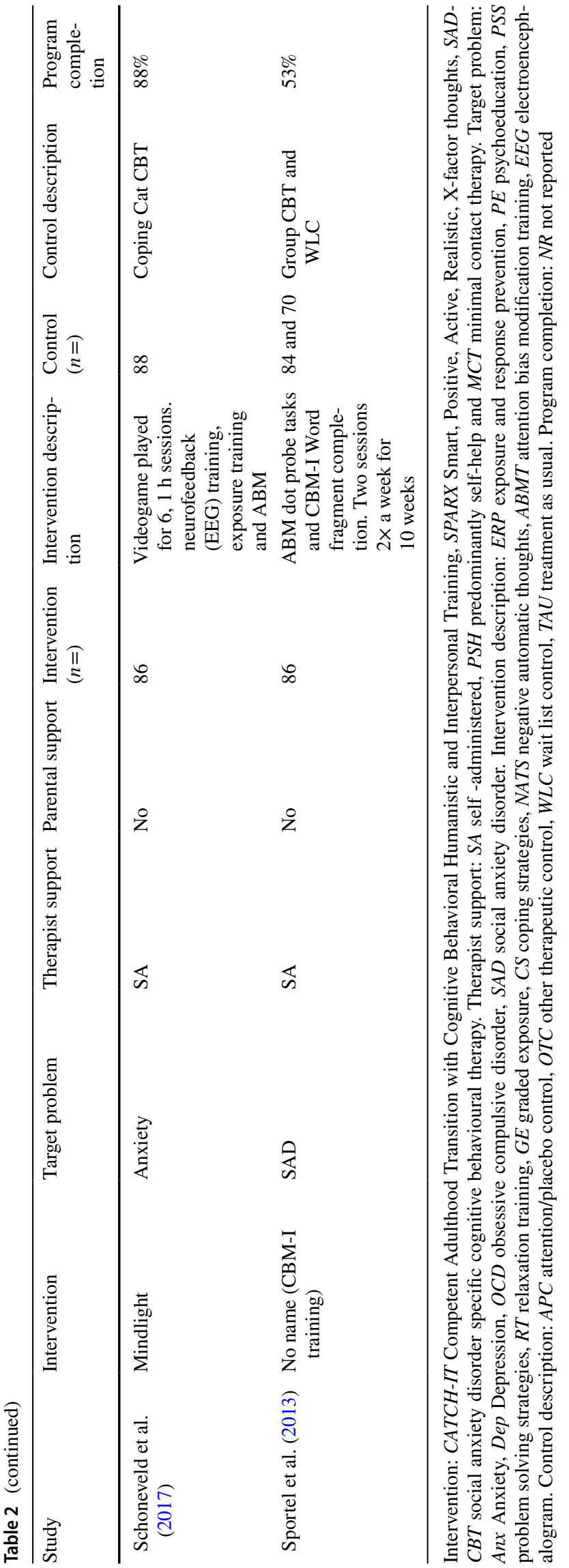

computer room at school. The Mindlight video game incorporates neurofeedback (EEG) training, exposure training, and attention bias modification training, played for 5-6 1-h sessions. Both trials involved school children with elevated anxiety symptoms. In one trial Mindlight was compared to a control game and in the other, to school-based CBT training based on the Coping - Cat program. None of the trials involved therapist guidance or parental support.

\section{Study Quality}

Study quality varied as demonstrated in Fig. 2. Most trials $(24 / 34,71 \%)$ adequately reported a random component in sequence generation, mostly using a computer-based random number generator. The remainder did not provide sufficient information to assess selection bias (10/34, 29\%). Most trials reported appropriate allocation concealment $(25 / 34,74 \%)$ meaning overall, the risk of selection bias was low. There appeared to be a high risk of performance bias as most of the studies could not ensure blinding of participants and personnel $(24 / 34,71 \%)$. Some studies told participants their group allocation in the first session while other studies were unable to ensure blinding due to the design of the study. Only 13/34 (38\%) studies reported adequate blinding of outcome assessment with the majority not providing enough information to assess (unclear risk, 18/34, 53\%). Overall, the risk of detection bias was therefore high. Risk of attrition bias tended to be low with the majority of studies using appropriate techniques to handle missing data $(25 / 34,74 \%)$. Only eight studies reported on a study protocol and were assigned low risk of reporting bias $(8 / 34,24 \%)$ with the remainder being assigned an unclear risk of reporting bias (26/34, 76\%). Finally, under half of the studies were assigned the low risk of 'other bias' $(15 / 34,44 \%)$.

\section{Program Completion}

Studies varied substantially in whether (and how) program completion rates were reported. Program completion was defined as completing all the modules/sessions in the intervention within the allotted study timeframe. For 22 studies $(65 \%)$ these data were clearly reported in the manuscript text or was extractable from CONSORT flow diagrams. For 12 studies $(35 \%)$ program completion was not reported or calculable from the provided information. Where reported, program completion rates ranged from 0 to $100 \%$ with $64 \%$ being the average program completion rate for intervention conditions.

\section{Meta-analysis}

A meta-analysis was undertaken to determine the effect of technology delivered interventions on depression and 
Random sequence generation (selection bias)

Allocation concealment (selection bias)

Blinding of participants and personnel (performance bias)

Blinding of outcome assessment (detection bias)

Incomplete outcome data (attrition bias)

Selective reporting (reporting bias)

Other bias
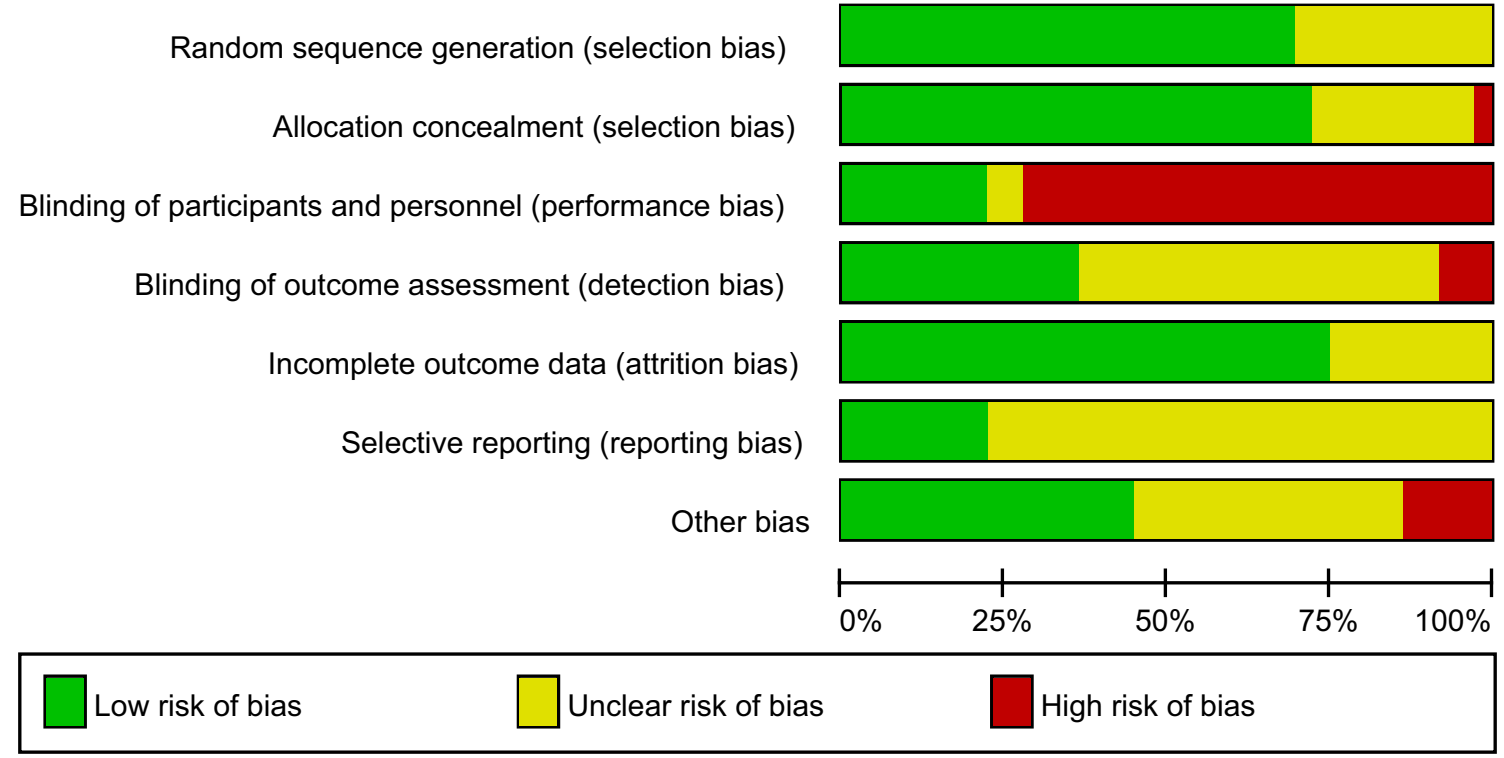

Fig. 2 Risk of bias graph. Review authors' judgements about each risk of bias item presented as percentages across all included studies

anxiety outcomes compared to wait-list control groups at post-intervention. A random-effects model produced a small overall effect in favor of technology delivered interventions: $g=0.45[95 \%$ CI $0.29,0.60]$. The associated $\mathrm{Z}$ score was significant $(Z=5.60, p<0.00001)$. Heterogeneity was high and statistically significant $\left(I^{2}=73 \%, Q=120.77, d f=33\right.$, $p<0.00001)$. Figure 3 is a forest plot of these results. The associated funnel plot (Fig. 4) is slightly asymmetrical, indicating possible publication bias.

\section{Sub-group Analyses}

\section{Age}

A sub-group analysis was conducted to determine whether effect sizes varied according to participant age. This analysis showed no significant difference ( $Q=0.36, d f=2, p=0.84$ ) between studies exclusively focused on children $(n=3$, $g=0.58,95 \%$ CI -0.01 to $1.18, p=0.05$ ), adolescent only samples $(n=18, g=0.42,95 \%$ CI $0.21-0.64, p<0.001)$, or mixed samples $(n=13, g=0.39,95 \%$ CI $0.15-0.63$, $p<0.001)$.

\section{Control Condition}

A sub-group analysis was performed to examine whether effect sizes varied according to the type of control group the intervention was compared to. There was a statistically significant difference $(Q=20.70, d f=3, p<0.001)$ in effect sizes according to category of control group. Technology-based interventions did not produce statistically significant benefits over face to face CBT interventions
( $n=4, g=0.11[-0.06$ to 0.28$] p=0.92$ ) or other therapy control conditions $(n=3, g=0.07$ [ -0.15 to 0.30$]$, $p=0.52)$. Technology-based interventions produced a small effect size demonstrating benefit over attention and placebo controls $(n=14, \mathrm{~g}=0.29$ [0.05-0.53], $p=0.02$ ) and a medium effect size demonstrating benefit compared to wait-list controls $(n=17, g=0.68$ [0.47-0.90], $p \leq 0.001)$.

\section{Mental Health Problem}

The following sub-group analysis investigated whether effect sizes differed according to the type of mental health problem, depression or anxiety. There was no significant difference $(Q=0.04, d f=1, p=0.83)$ in effect sizes between interventions targeting depression $(n=13$, $g=0.43$ [95\% CI 0.18-0.68]) and interventions targeting anxiety $(n=213, g=0.41[95 \%$ CI $0.12-0.71])$.

\section{Problem Severity}

We explored whether diagnostic status influenced effect sizes. There was a significant difference $(Q=13.44, d f=1$, $p<0.001)$ between interventions involving participants with a primary diagnosis of depression or an anxiety disorder $(n=18, g=0.72[95 \%$ CI $0.52-0.91] p<0.001)$ and those involving participants with elevated symptoms of depression or anxiety $(n=16, g=0.22$ [95\% CI $0.03-0.40]$ $p=0.02$ ). Interventions that involved participants with diagnosed disorders had larger effect sizes. 


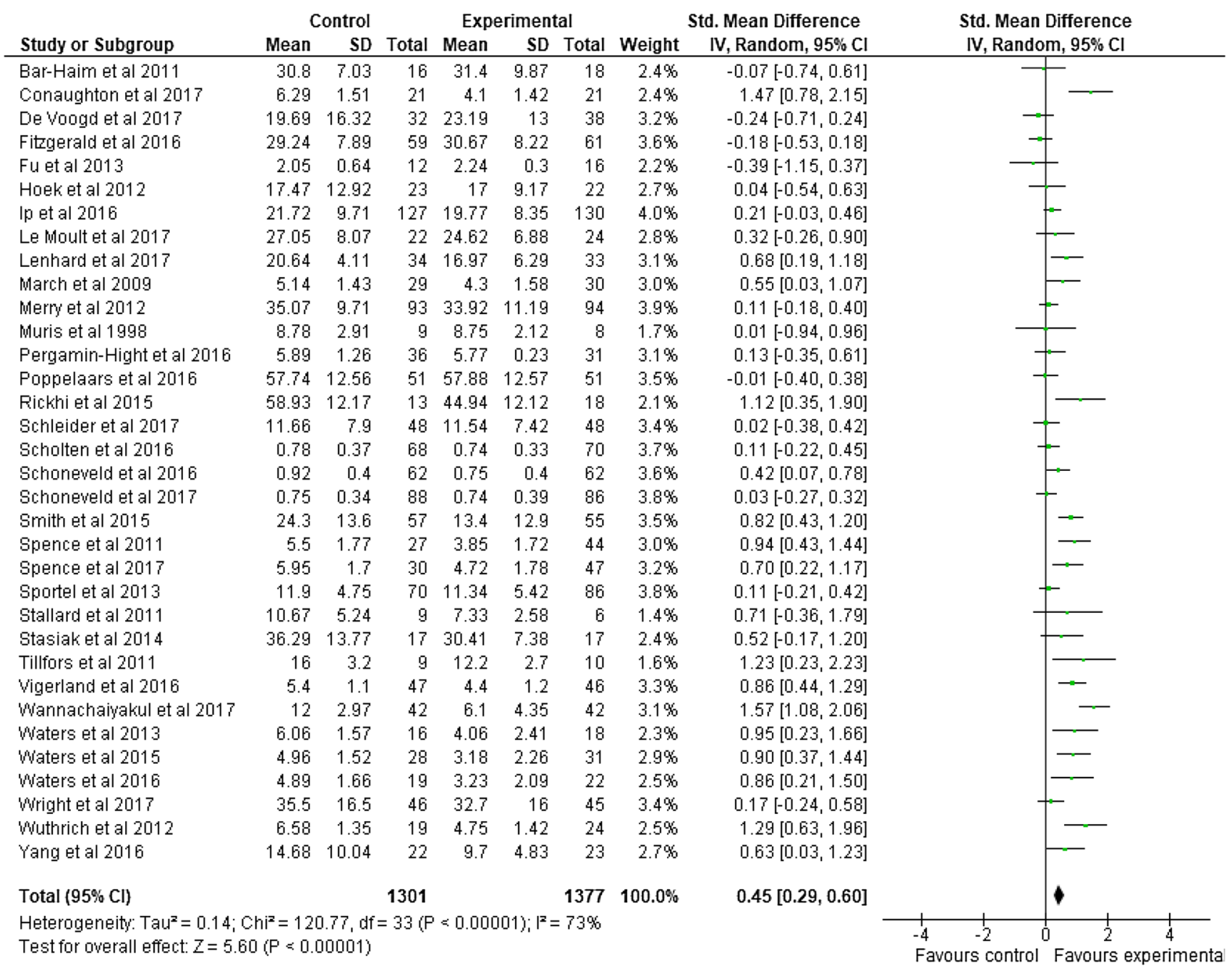

Fig. 3 Forest Plot of meta-analysis on technology delivered interventions for depression and anxiety in adolescents compared to control conditions

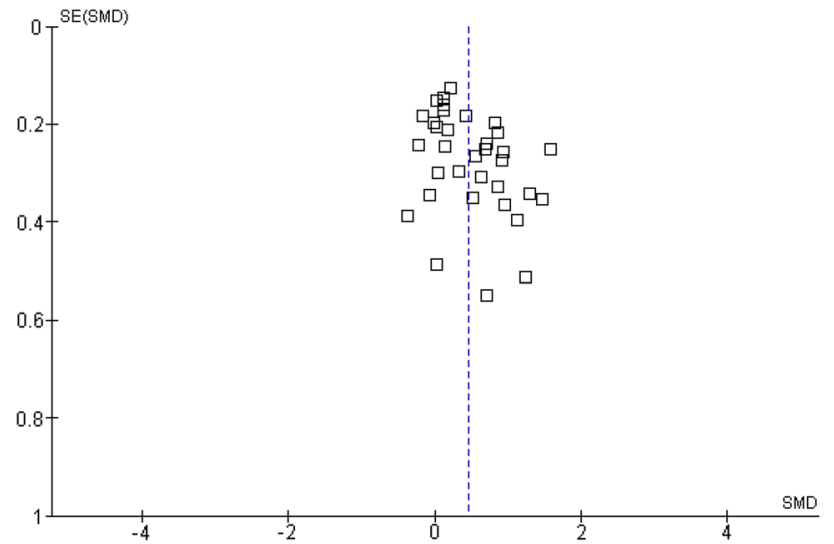

Fig. 4 Funnel Plot

\section{Theoretical Basis}

Studies were grouped according to the theoretical basis of the intervention, including CBT, ABMT, CBM and 'other'. Sub-group analysis demonstrated a statistically significant difference $(Q=11.61, d f=3, p=0.009)$ in effect sizes between interventions based on CBT $(n=17, g=0.66$ [95\% CI 0.42-0.90] $p<0.001)$, interventions based on ABMT $(n=8, g=0.41$ [95\% CI 0.08-0.73] $p=0.01)$, CBM interventions $(n=3, g=0.09$ [95\% CI -0.19-0.37] $p=0.53)$, and 'other' interventions $(n=6, g=0.20$ [95\% CI -0.03-0.44] $p=0.09$ ). Other interventions and CBM interventions did not demonstrate statistically significant benefits over control conditions. 


\section{Therapist Support}

We explored whether therapist support influenced effect sizes. There was a significant effect of therapist support on trial effect sizes $(Q=27.28, d f=2, p<0.001)$. Minimal contact therapy produced larger effect sizes $(n=9, g=0.87$ [95\% CI $0.68,1.06] p<0.001)$, than predominantly selfhelp $(n=2, g=0.81[-0.68,2.31] p=0.29)$ and purely self-administered interventions $(n=23, g=0.24[0.10,0.38]$, $p<0.001)$.

\section{Parental Involvement}

The next sub-group analysis investigated whether parental support of the intervention influenced effect sizes. Results showed a statistically significant difference $(Q=24.43$, $d f=1, p<0.001)$ with parent supported interventions producing larger effect sizes $(n=9, g=0.86$ [95\% CI 0.69, 1.04] $p<0.001)$ than interventions delivered without parent support $(n=23, g=0.25$ [95\% CI 0.09, 0.42] $p=0.002)$.

\section{Continuation of Other Treatment}

Finally, we explored whether continuation of other treatment for depression or anxiety influenced effect sizes. Results demonstrated a significant difference in effect sizes $(Q=9.37, d f=1, p=0.002)$ between trials in which continuation of psychological or pharmacological treatment was permitted ( $n=6, g=0.90$ [95\% CI 0.68, 1.11], $p<0.001$ ) and trials in which no ongoing treatment was provided ( $n=16, g=0.42$ [95\% CI $0.20,0.63]$ ). The provision of ongoing treatment generated larger effect sizes than trials in which ongoing treatment was not permitted. Some studies were excluded from this analysis due to this information not being reported $(n=12)$.

\section{Discussion}

The aim of this study was to provide an up-to-date investigation of the effect of technology delivered interventions for the treatment of depression and anxiety in children and adolescents. Our systematic search identified 34 randomized controlled trials involving 3113 children and adolescents aged 6-18 years of age. Our search failed to identify any studies of emerging technologies such as virtual reality and m-Health applications (apps) developed specifically for children and adolescents with depression and anxiety. Whilst our search results indicate that research in this area is growing, research does not appear to be keeping pace with advances in technological development. Similarly, the 34 studies we identified related to 29 different programs. Nearly all were, therefore, subject to a single evaluation undertaken by the program developer. Where additional evaluations were undertaken (four programs) these had typically been undertaken with the involvement of the program developers highlighting the need for further independent evaluation.

Technological interventions based on CBT programs yielded a medium post-intervention effect compared to waiting list control groups. This highlights the benefit of technology-based interventions for depression and anxiety in children and adolescents. Given the limited capacity and long waiting times for appointments in many specialist child and adolescent mental health services, technological interventions could offer an effective way of increasing timely access to evidence-based interventions. The majority of these interventions required minimal therapist support and could readily be provided for those on long waiting lists.

\section{Comparison with Previous Work}

CBT based technology delivered interventions yielded an effect size $(g=0.66)$ comparable to other meta-analyses which have shown moderate effect sizes for face to face CBT in children ( $d=0.66$; Arnberg and Öst 2014) and for CCBT/ICBT for adolescents ( $g=0.72$; Ebert et al. 2015). Although ABMT based interventions produced a small effect size $(g=0.41)$ this is larger than those reported in previous reviews $(g=-0.19$, Pennant et al. 2015). As a general update on the research literature since the work of Pennant et al. (2015), our review has found 21 new RCT's published since 2013. In accordance with Pennant et al's work, we mostly found CCBT/ICBT and ABMT based programs and failed to find any new RCT's of Smartphone apps or virtual reality-based programs. Pennant et al.could not be confident in the effect of CCBT on children (defined as 5-11 years). In the present analysis, we demonstrated no significant difference in effect sizes between studies with children (5-11 years), adolescents (12-18 years) and mixed ages (5-18 years) and all sub-groups demonstrated a significant benefit over control groups. However, we only identified three studies involving a total of 67 children which had an exclusive child sample (Bar-Haim et al. 2011; Waters et al. 2015, 2016). Of these, two studies also permitted ongoing treatment and compared ABMT to a wait-list control, factors which in our review significantly moderated outcomes. The evidence to support the use of technological interventions with children under the age of 12 remains limited and as such we cannot be confident in the effects of these interventions with children. Further research is required to investigate the effects of technological interventions on children and the level of parental support that may be beneficial. 


\section{Sub-group Analyses Findings}

Technology delivered interventions failed to demonstrate a significant benefit over face to face CBT or other therapy conditions. This finding is in accordance with a previous meta-analysis of ICBT in children and adolescents. This also found no significant difference in effects between ICBT and face to face CBT (Vigerland et al. 2016b). Like Vigerland et al. (2016b) our analysis only includes a small number of studies utilizing face to face CBT as a control (four) which may limit our conclusions. It is also important to note that two out of the four interventions were not based on CBT. Mindlight (Schoneveld et al. 2017) is a videogame incorporating neurofeedback (EEG) training, exposure training and $\mathrm{ABM}$; the other intervention is based on cognitive bias modification (Sportel et al. 2013). Both these approaches have a limited evidence base, particularly in comparison to face to face CBT. In contrast, face to face CBT is not only supported by a large evidence base (Hofmann et al. 2012) but is also considered to be the 'gold standard' in psychological treatment (David et al. 2018).

Comparing a technology-based intervention to an attention placebo control produced statistically significant benefits and a small effect size. Comparing technology delivered interventions to wait-list control groups yielded a moderate effect in favor of the intervention. This is consistent with previous analyses which have demonstrated moderate effects using this comparison in adolescent populations (Richards and Richardson 2012; Vigerland et al. 2016b), in adult populations (Grist and Cavanagh 2013) and in comparing face to face CBT with wait-list groups (Hofmann et al. 2012). As such our conclusions are similar to those of Pennant et al. (2015); that evidence for the benefit of technology-based interventions compared to face to face therapies is sparse and should not replace face to face therapy. It does however suggest that technological interventions could offer a lowcost alternative treatment when face to face treatments are not available or feasible.

There was no significant difference in effect sizes between interventions targeting depression and those targeting anxiety, suggesting programs for depression and anxiety were equally effective. In a recent meta-review of digital health interventions for children and young people, Hollis et al. (2017) summarized findings from six meta-analyses (Davies et al. 2014; Ebert et al. 2015; Pennant et al. 2015; Podina et al. 2016; Ye et al. 2014) which demonstrated CCBT for depression yielded small to moderate effect sizes, whereas CCBT for anxiety yielded moderate to large effects in favor of the intervention. Hollis et al. (2017) did not, however, analyze whether these differences were significantly different. The results from the present analysis would suggest they may not be statistically, or meaningfully different.
Sub-group analyses also indicated that technological interventions involving participants with a confirmed diagnosis of depression or anxiety produced significantly larger effect sizes than interventions trialed with populations experiencing elevated symptoms. Few previous meta-analyses have explored the effect of problem severity on effect sizes and those that have, yield contrasting results. Ebert et al's (2015) analysis found no significant difference in effect sizes between trials involving participants (up to age 25) with a 'confirmed diagnosis' $(n=6 ; g=0.71)$ and those with 'anxiety/depressive symptoms' $(n=7, g=0.74)$ although this focused only on computer and internet CBT. The analysis by Pennant et al. (2015) found a significant effect for anxiety but not for depression although the authors note that sample sizes were small. Our findings contrast with these reviews but are consistent with findings involving adults. Analyses of outcome predictors for iCBT for depression in adults have demonstrated that higher pre-treatment symptom severity is related to greater symptom reduction (Button et al. 2012; Edmonds et al. 2018). This finding tends to be explained in terms of individuals with greater symptom severity having greater room for improvement and symptom reduction (Edmonds et al. 2018). This may explain why effect sizes in our analysis were larger for diagnosed groups. Future research should explore the role of problem severity in treatment outcomes to determine for whom technology delivered interventions will be beneficial.

Our review found that interventions based on CBT produced the largest effect sizes, followed by interventions based on ABMT. The potential benefit of CBT based interventions adds to the conclusions of previous reviews of ICBT/CCBT (Ebert et al. 2015; Pennant et al. 2015; Podina et al. 2016; Vigerland et al. 2016a) and is expected given the developed evidence-based for CBT as an intervention for depression and anxiety (Cuijpers et al. 2013; Watts et al. 2015). As noted previously, CBT is considered the 'gold standard' psychological therapy (David et al. 2018). Interestingly, our finding that ABMT produced small, significant effects diverges from the findings of Pennant et al. (2015) for whom three ABMT studies produced no significant benefit over control conditions. However, our conclusions remain similar; while there is some indication that ABMT may be effective, we cannot be confident in the demonstrated benefit of ABMT as studies and the overall effect sizes were small. Both CBM based interventions and interventions classified as 'other' (problem-solving therapy, growth mindset, bio/ neurofeedback emotion regulation videogames) failed to demonstrate a significant benefit over control conditions. This may reflect the limited research which has been undertaken but at this stage, we cannot be confident in the benefit of these interventions.

Previous evidence demonstrates mixed results regarding the role of therapeutic support in e-Health interventions. 
In adult populations, meta-analyses consistently demonstrate that therapist supported programs produce larger effect sizes than programs that provide no therapist support (Johansson and Andersson 2012; Richards and Richardson 2012). For children and adolescents, findings from Pennant et al. (2015) indicated no significant difference between programs for depression with 'minimal' vs. 'some' therapist input. Other evidence suggests that for programs targeting anxiety, 'minimally' supported interventions yield larger effect sizes than 'significantly' supported programs (Podina et al. 2016). Our analysis found that effect size was related to therapeutic support, specifically, minimal contact therapies (more than $90 \mathrm{~min}$ ) yielded higher effect sizes than purely self-administered interventions. In traditional face to face therapies, a body of research has supported the role of 'common factors', such as the working alliance, in producing positive therapeutic outcomes (Lambert 1992). It is logical therefore that the more opportunity for interaction there is with a therapist in technology-based interventions, the more scope there is to build a therapeutic relationship, thereby improving outcomes. Therapeutic support has also been identified as a moderating factor influencing engagement as well as therapeutic outcomes (Hollis et al. 2017; Rickwood and Bradford 2012). Therefore, therapeutic support is also likely to improve engagement and motivation to continue using technology-based interventions. These findings extend those of Richards and Richardson (2012) to a child and adolescent population.

The importance of parental support in CCBT and technology delivered interventions is poorly understood (Vigerland et al. 2016b). The parental input may range from assisting children and adolescents in setting up the program to active participation in program sessions. Our sub-group analysis showed parent supported interventions produced significantly larger effect sizes than interventions delivered without, although both types of programs were significantly better than control groups. These results contrast with those of Ebert et al. (2015). These divergent results may be explained by the differences in study eligibility criteria previously described. Our results do however corroborate previous work with face to face CBT in children which suggests treatment outcomes can be improved with parental involvement (Barrett et al. 1996; Creswell and Cartwright-Hatton 2007). It has been suggested that parental involvement may aid the successful learning and application of new skills, perspectives and applying specific strategies learned in therapy into real life (Siddaway et al. 2014; Spence et al. 2000; Stallard 2005; Thirlwall et al. 2013). As such their children might be more likely to continue to understand and implement therapeutic strategies and so make therapeutic gains (Thirlwall et al. 2013). Parental involvement may also aid changes in family processes and parental risk factors implicated in the development and maintenance of childhood anxiety (Barrett et al. 1996; Siddaway et al. 2014).

As far as we are aware, no previous meta-analysis of e-Health in children and adolescents with depression or anxiety has explored whether continuation of other psychological or pharmacological treatment for depression or anxiety during the trial influences effect sizes. Our sub-group analysis demonstrated significant differences between trials permitting ongoing treatment and those explicitly forbidding ongoing psychological and/or pharmacological treatment. Trials permitting ongoing treatment produced a large effect size, whereas trials forbidding ongoing treatment yielded a small effect size, both significant. It is possible that for the ongoing treatment permitted studies, the larger effect sizes are due to a 'combination effect' of the program, plus any ongoing treatment, or they could be due to either the program or ongoing intervention alone. While some previous work has shown that 'combined treatment' is superior to psychological interventions or pharmacological interventions in adults (Cuijpers et al. 2014), for children this is uncertain (Cox et al. 2014; Walkup et al. 2008). It is important to acknowledge that only three out of the six studies (Lenhard et al. 2017; Rickhi et al. 2015; Smith et al. 2015) actually reported the number of participants who received an ongoing psychological or pharmacological treatment during the study intervention. The remaining three only noted in the inclusion criteria that ongoing treatment was permitted (Tilfors et al. 2011; Vigerland et al. 2016a; Wuthrich et al. 2012). Therefore, we cannot be certain of the extent or nature of the additional ongoing treatment that was provided. Our conclusions about the benefits of using technology alongside ongoing interventions requires further exploration and should be interpreted cautiously.

Finally, as with previous reviews (Ebert et al. 2015; Pennant et al. 2015), most RCT's were conducted in highincome countries and we cannot, therefore, generalize these results to low- or middle-income countries. The need for access to evidence-based mental health interventions in low-income countries is considerable and the potential of technology delivered interventions in meeting this need will not be fulfilled until future research is conducted in these contexts.

\section{Limitations}

While our results demonstrate the potential benefit of technology delivered interventions, our review does have a number of limitations: First, our analyses are limited to postintervention outcomes only and we were unable to assess whether these immediate benefits persist into the medium term. Second, our main analysis compared technological interventions with wait-list control groups assessed after a specified period of time. Whilst this provides a consistent 
timeframe for comparisons some participants may not have completed the intervention by follow-up. Reporting of program completion rates was poor and were unavailable for 12 studies. Where we were able to extract them the average program completion rate of $64 \%$ indicates that a number of participants had not completed the program. Third, the included studies suffered from several methodological limitations. Study protocols were seldom reported, and sample sizes were small with 14 reporting a sample of fewer than 50 participants. Although there was significant bias regarding the non-blinding of participants this is inevitable when comparing an intervention to a waiting list condition. Ethical practice requires participants to be fully aware of the study conditions and as such it is impossible to effectively blind participants to treatment allocation (Button et al. 2015). Finally, our search strategy was limited to published papers. Given the speed of growth in this area, it is probable that further studies might have been identified.

\section{Future Directions}

While the presented studies offer an insight into the potential benefit of technology delivered programs, future work requires better quality trial design and reporting; the use of adequate sample sizes, appropriate active control groups as well as adequate reporting of program completion and attrition. Well-designed RCT's will also be required to determine the sole effect of ICBT/CCBT distinct from combined effects with ongoing psychological or pharmacological treatment. Adequate investigation and reporting of the costeffectiveness of technology-based interventions are also necessary, particularly because cost-effectiveness is one of the main proposed benefits of technology-based interventions (Vigerland et al. 2016b).

As far as can be determined from the information reported, none of the programs trialed included adolescents in program design. Recent work investigating a m-Health intervention for children and adolescents who self-harm (Stallard et al. 2018) demonstrated the value of involvement of adolescents with lived experience in the design of interventions, particularly for program acceptability. Given the high level of attrition from e-Health interventions (Melville et al. 2010) it would be beneficial for future work to explore the impact of program co-production on engagement and attrition.

\section{Conclusions}

This meta-analysis provides a unique update on the current evidence for the effect of technology-based interventions in children and adolescents. Our systematic search identified
34 RCTs examining technology delivered interventions (primarily based on CBT and ABMT) for depression or anxiety in youth populations. Overall, the analysis provides support for the effectiveness of CBT based technology delivered interventions for both depression and anxiety in comparison to wait-list controls. Interventions based on ABMT yielded only a small effect size and CBM and 'other' programs (problem-solving therapy, growth mindset, bio/neurofeedback emotion regulation videogames) failed to demonstrate a significant benefit over control groups. We, therefore, cannot be confident in the benefit of these interventions at this stage.

Therapist supported, and parent supported programs produced better outcomes. Given that therapist-assisted programs produced better outcomes and comparisons to face to face CBT did not confer any significant benefit, our overall conclusions are consistent with previous work (Hollis et al. 2017; Pennant et al. 2015): The current evidence base does not support the use of technology delivered interventions as a replacement for face to face psychological interventions. However, the magnitude of effects demonstrated suggest there is a benefit in using technology delivered CBT interventions where access to face to face CBT and other psychotherapies are limited or delayed.

Funding This research did not receive any specific grant from funding agencies in the public, commercial, or not-for-profit sectors.

\section{Compliance with Ethical Standards}

Conflict of interest The authors declare that they have no conflict of interest.

Ethical Approval All procedures performed in studies involving human participants were in accordance with the ethical standards of the institutional and/or national research committee and with the 1964 Helsinki declaration and its later amendments or comparable ethical standards.

Open Access This article is distributed under the terms of the Creative Commons Attribution 4.0 International License (http://creativeco mmons.org/licenses/by/4.0/), which permits unrestricted use, distribution, and reproduction in any medium, provided you give appropriate credit to the original author(s) and the source, provide a link to the Creative Commons license, and indicate if changes were made.

\section{Appendix A: Search Strings by Databases}

\author{
APA PsychNet
}

27/09/17

254 Results for Any Field: CCBT OR Any Field: "computer assisted therapy" OR Any Field: computerised OR Any Field: computerized OR Any Field: computer OR Any Field: "CD-ROM" OR Any Field: "DVD-ROM" OR Any 
Field: "mHealth*" OR Any Field: "mobile health" OR Any Field: "mobile device" OR Any Field: "mobile app" OR Any Field: "smartphone" OR Any Field: "mobile phone" OR Any Field: Internet OR Any Field: "ICBT" OR Any Field: web OR Any Field: "virtual reality" AND Any Field: "depress*" OR Any Field: "self - harm" OR Any Field: "suicid*" OR Any Field: anxi* OR Any Field: "PTSD" OR Any Field: "social anxi*" OR Any Field: "separation anxi*" OR Any Field: phobia OR Any Field: "generalised anxiety disorder" OR Any Field: "OCD" AND Any Field: child* OR Any Field: teenage* OR Any Field: adolescen* OR Any Field: "young per*" OR Any Field: youth* AND Any Field: "randomized controlled trial" OR Any Field: randomized OR Any Field: randomised AND Age Group: Preschool Age (2-5 yrs) OR School Age (6-12 yrs) OR Adolescence (1317 yrs) AND Document Type: Journal Article AND Year: 2013 To 2018

Embase

27/09/17

189 results for search (ccbt OR 'computer assisted therapy'/exp OR 'computer assisted therapy' OR computerised OR computerized OR 'computer'/exp OR computer OR 'cd-rom'/exp OR 'cd-rom' OR 'dvd-rom' OR mhealth* OR 'm-health*' OR 'mobile health'/exp OR 'mobile health' OR 'mobile device'/exp OR 'mobile device' OR 'mobile app'/ exp OR 'mobile app' OR 'smartphone'/exp OR smartphone OR 'mobile phone'/exp OR 'mobile phone' OR 'internet'/ exp OR internet OR icbt OR 'web'/exp OR web OR vr OR 'virtual reality'/exp OR 'virtual reality') AND (depress* OR 'self-harm' OR suicid* OR anxi* OR 'ptsd'/exp OR ptsd OR 'social anxi*' OR 'separation anxi*' OR 'phobia'/ exp OR phobia OR 'generalised anxiety disorder'/exp OR 'generalised anxiety disorder' OR ocd) AND (child* OR teenage* OR adolescen* OR 'young per*' OR youth* OR 'young adult*') AND ('randomized controlled trial'/exp OR 'randomized controlled trial' OR randomised) AND [2013-2017]/py AND 'randomized controlled trial'/de AND 'article'/it AND ([adolescent]/lim OR [child]/lim OR [preschool]/lim OR [school]/lim)

Google Scholar

27/09/17

31 results for Computerized CCBT mHealth "smartphone app" "virtual reality" "mental health" child adolescent "randomized controlled trial"

PubMed

27/09/17

254 results for

((( (ccbt OR "computer assisted therapy" OR computerized OR computerized OR computer OR cd-rom OR dvdrom OR "mHealth*" OR "m-health*" OR "mobile health" OR "mobile device" OR "mobile app" OR "smartphone" OR "mobile phone" OR internet OR icbt OR web OR "virtual reality")) AND (“depress*” OR "self - harm" OR suicid* OR anxi* OR "PTSD" OR "social anxi*” OR "separation anxi*" OR phobia OR "generalised anxiety disorder" OR “OCD”)) AND (child* OR teenage* OR adolescen* OR "young per*" OR youth*)) AND ("randomized controlled trial" OR randomized OR randomised) Filters activated: Publication date from 2013/01/01 to 2018/12/31, Child: birth-18 years

\section{Science Direct}

Search results: 67 results found for pub-date $>2012$ and TITLE-ABSTR-KEY(Computerized OR CCBT OR mHealth OR "smartphone app" OR "virtual reality") and ("mental health" AND child OR adolescent AND "randomized controlled trial").

\section{Scopus}

27/09/17

909 results for

(ccbt OR "computer assisted therapy" OR computerised OR computerized OR computer OR cd-rom OR dvd-rom OR "mHealth*" OR "m-health*" OR "mobile health" OR "mobile device" OR "mobile app" OR "smartphone" OR "mobile phone" OR internet OR icbt OR web OR "virtual reality") AND ("depress*" OR "self -harm" OR suicid* OR anxi* OR "PTSD” OR "social anxi*” OR "separation anxi*" OR phobia OR "generalised anxiety disorder" OR "OCD") AND (child* OR teenage* OR adolescen* OR "young per*" OR youth* OR "young adult*") AND ("randomized controlled trial") AND (LIMIT-TO (SRCTYPE, “j ")) AND (LIMIT-TO (DOCTYPE, "ar ")) AND (LIMITTO (SUBJAREA, "PSYC ")) AND (LIMIT-TO (PUBYEAR, 2018) OR LIMIT-TO (PUBYEAR, 2017) OR LIMIT-TO (PUBYEAR, 2016) OR LIMIT-TO (PUBYEAR, 2015) OR LIMIT-TO (PUBYEAR, 2014) OR LIMIT-TO (PUBYEAR, 2013)) AND (LIMIT-TO (LANGUAGE, "English ")) AND (LIMIT-TO (EXACTKEYWORD, "Controlled Study ") OR LIMIT-TO (EXACTKEYWORD, "Adolescent ")) AND (LIMIT-TO (EXACTKEYWORD, "Adolescent"))

\section{Social Policy and Practice}

\section{7 results}

27/09/2016

(CCBT or "computer assisted therapy" or computerised or computerized or computer or CD-ROM or DVD-ROM or mHealth or "m-health" or "mobile health" or "mobile device" or "mobile app" or smartphone or "mobile phone" or Internet or ICBT or web or "virtual reality") and (depression or "self-harm" or suicide or anxiety or "PTSD" or "social anxiety" or "separation anxiety OR phobia" or "generalised anxiety disorder" or "OCD" or "conduct disorder" or "eating disorder" or anorexia or bulimia or "binge eating" or "body image" or "mental health" or schizophrenia or "bipolar affective disorder" or psychosis or insomnia or ADHD or Autism or substance) and (child* or teenage* or adolescent or "young person" or youth* or "young adult") 
and ("randomized controlled trial" or randomized or randomised)).af. limit 1 to $y r=$ "2013-Current"

\section{Web of Science}

\section{7/09/2017}

\section{Results: 361}

\section{(from Web of Science Core Collection)}

You searched for: TOPIC: (CCBT OR computer assisted therapy OR Computerised OR computerized OR computer OR CD-ROM OR DVD-ROM OR mHealth* OR m-health* OR mobile health OR mobile device OR mobile app OR smartphone OR mobile phone OR Internet OR ICBT OR web OR virtual reality) ANDTOPIC: (depress* OR self harm OR suicid* OR anxi* OR PTSD OR social anxi* OR separation anxi*OR phobia OR generalised anxiety disorder OR OCD) AND TOPIC: (child* OR teenage* OR adolescen* OR young per* OR youth*) $A N D$ TOPIC:("randomized controlled trial")

Timespan: 2013-2017. Indexes: SCI-EXPANDED, SSCI, A\&HCI, ESCI.

\section{JMIR}

\section{$27 / 09 / 17$}

33 results

Abstract Computerized CCBT mHealth "smartphone app" "virtual reality" All fields depression OR anxiety AND child adolescent AND "randomized controlled trial"

Cyberpsychology, Behavior and Social Networking 26/09/17

\section{RESULTS}

You searched for: [[All: "computerized"] OR [All: ccbt] OR [All: mhealth] OR [All: "smartphone]] AND [[All: app"]OR [All: "virtual]] AND [All: reality"] AND [All: "mental health"] AND [[All: child] OR [All: adolescent]] AND [Publication Date: (01/01/2013 TO 12/31/2017)] AND [[Categories: Psychology, Humanities, and Social Science] OR [Book/Issue: Advances in Preschool Psychopharmacology] OR [in Journal: Journal of Child and Adolescent Psychopharmacology] OR [in Journal: Violence and Gender] OR [in Journal: Cyberpsychology, Behavior, and Social Networking]]

6 articles matched your search criteria.

\section{References}

Andersson, G., \& Cuijpers, P. (2009). Internet-based and other computerized psychological treatments for adult depression: a metaanalysis. Cognitive Behaviour Therapy, 38(4), 196-205.

Andrews, G., Cuijpers, P., Craske, M. G., McEvoy, P., \& Titov, N. (2010). Computer therapy for the anxiety and depressive disorders is effective, acceptable and practical health care: A meta-analyss. PLOS ONE, 5(10), e13196.

Arnberg, A., \& Öst, L. G. (2014). CBT for children with depressive symptoms: A meta-analysis. Cognitive Behaviour Therapy, 43(4), 275-288
Barrett, P. M., Dadds, M. R., \& Rapee, R. M. (1996). Family treatment of childhood anxiety: A controlled trial. Journal of Consulting and Clinical Psychology, 64(2), 333.

Birmaher, B., Ryan, N. D., Williamson, D. E., Brent, D. A., Kaufman, J., Dahl, R. E., et al. (1996). Childhood and adolescent depression: A review of the past 10 years. Part I. Journal of the American Academy of Child \& Adolescent Psychiatry, 35(11), 1427-1439.

Boydell, K. M., Hodgins, M., Pignatiello, A., Teshima, J., Edwards, H., \& Willis, D. (2014). Using technology to deliver mental health services to children and youth: A scoping review. Journal of the Canadian Academy of Child and Adolescent Psychiatry, 23(2), 87.

Button, K. S., Wiles, N. J., Lewis, G., Peters, T. J., \& Kessler, D. (2012). Factors associated with differential response to online cognitive behavioural therapy. Social Psychiatry and Psychiatric Epidemiology, 47(5), 827-833.

Calear, A. L., \& Christensen, H. (2010). Review of internet-based prevention and treatment programs for anxiety and depression in children and adolescents. Medical Journal of Australia, 192(11), S12.

Clarke, A. M., Kuosmanen, T., \& Barry, M. M. (2015). A systematic review of online youth mental health promotion and prevention interventions. Journal of youth and adolescence, 44(1), 90-113.

Costello, E. J., Erkanli, A., \& Angold, A. (2006). Is there an epidemic of child or adolescent depression? Journal of Child Psychology and Psychiatry, 47(12), 1263-1271.

Cox, G. R., Callahan, P., Churchill, R., Hunot, V., Merry, S. N., Parker, A. G., et al. (2014). Psychological therapies versus antidepressant medication, alone and in combination for depression in children and adolescents. The Cochrane Library, 11, CD008324

Crombie, I., \& Davies, H. (2009). What is meta-analysis?. London: Hayward Medical Communications.

Cuijpers, P., Berking, M., Andersson, G., Quigley, L., Kleiboer, A., \& Dobson, K. S. (2013). A meta-analysis of cognitive-behavioural therapy for adult depression, alone and in comparison with other treatments. The Canadian Journal of Psychiatry, 58(7), 376-385.

Cuijpers, P., Sijbrandij, M., Koole, S. L., Andersson, G., Beekman, A. T., \& Reynolds, C. F. (2014). Adding psychotherapy to antidepressant medication in depression and anxiety disorders: A metaanalysis. World Psychiatry, 13(1), 56-67. https://doi.org/10.1002/ wps.20089.

David, D., Cristea, I., \& Hofmann, S. G. (2018). Why cognitive behavioral therapy is the current gold standard of psychotherapy. Frontiers in Psychiatry, 9, 4. https://doi.org/10.3389/fpsyt.2018.00004.

Davies, E. B., Morriss, R., \& Glazebrook, C. (2014). Computer delivered and web-based interventions to improve depression, anxiety, and psychological well-being of university students: A systematic review and meta-analysis. Journal of Medical Internet Research, 16, e130.

Ebert, D. D., Zarski, A. C., Christensen, H., Stikkelbroek, Y., Cuijpers, P., Berking, M., et al. (2015). Internet and computer-based cognitive behavioral therapy for anxiety and depression in youth: a meta-analysis of randomized controlled outcome trials. PLoS ONE, 10(3), e0119895.

Edmonds, M., Hadjistavropoulos, H. D., Schneider, L. H., Dear, B. F., \& Titov, N. (2018). Who benefits most from therapist-assisted internet-delivered cognitive behaviour therapy in clinical practice? Predictors of symptom change and dropout. Journal of Anxiety Disorders, 54, 24-32.

Essau, C. A., Conradt, J., Sasagawa, S., \& Ollendick, T. H. (2012). Prevention of anxiety symptoms in children: Results from a universal school-based trial. Behavior Therapy, 43(2), 450-464.

Fletcher, J. M. (2008). Adolescent depression: Diagnosis, treatment, and educational attainment. Health Economics, 17(11), $1215-1235$.

Fonagy, P., Pugh, K., \& O'Herlihy, A. (2017). The Children and Young People's Improving Access to Psychological Therapies (CYP IAPT) Programme in England. In D. Skuse, H. Bruce \& L. 
Dowdney (Eds.), Child Psychology and Psychiatry. https://doi. org/10.1002/9781119170235.ch48.

Ford, T., Hamilton, H., Goodman, R., \& Meltzer, H. (2005). Service contacts among the children participating in the British child and adolescent mental health surveys. Child and Adolescent Mental Health, 10(1), 2-9.

Gould, M. S., Greenberg, T. E. D., Velting, D. M., \& Shaffer, D. (2003). Youth suicide risk and preventive interventions: A review of the past 10 years. Journal of the American Academy of Child \& Adolescent Psychiatry, 42(4), 386-405.

Griffiths, K. M., Farrer, L., \& Christensen, H. (2010). The efficacy of internet interventions for depression and anxiety disorders: A review of randomised controlled trials. Medical Journal of Australia, 192(11), S4.

Grist, R., \& Cavanagh, K. (2013). Computerised cognitive behavioural therapy for common mental health disorders: What works and for whom? A systematic review and meta-analysis. Journal of Contemporary Psychotherapy, 43(4), 243-251.

Hofmann, S. G., Asnaani, A., Vonk, I. J. J., Sawyer, A. T., \& Fang, A. (2012). The efficacy of cognitive behavioral therapy: A review of meta-analyses. Cognitive Therapy and Research, 36(5), 427-440. https://doi.org/10.1007/s10608-012-9476-1.

Hollis, C., Falconer, C. J., Martin, J. L., Whittington, C., Stockton, S., Glazebrook, C., et al. (2017). Annual Research Review: Digital health interventions for children and young people with mental health problems - A systematic and meta-review. Journal of Child Psychology and Psychiatry, 58(4), 474-503.

James, A. C., James, G., Cowdrey, F. A., Soler, A., \& Choke, A. (2013). Cognitive behavioural therapy for anxiety disorders in children and adolescents. Cochrane Database of Systematic Review. https ://doi.org/10.1002/14651858.CD004690.pub3

Johansson, R., \& Andersson, G. (2012). Internet-based psychological treatments for depression. Expert Review of Neurotherapeutics, $12,861-870$..

Johnson, K. R., Fuchs, E., Horvath, K. J., \& Scal, P. (2015). Distressed and looking for help: Internet intervention support for Arthritis self-management. Journal of Adolescent Health, 56(6), 666-671.

Kim-Cohen, J., Caspi, A., Moffitt, T. E., Harrington, H., Milne, B. J., \& Poulton, R. (2003). Prior juvenile diagnoses in adults with mental disorder: Developmental follow-back of a prospective longitudinal cohort. Archives of General Psychiatry, 60(7), 709-717.

Lal, S., \& Adair, C. E. (2014). E-mental health: A rapid review of the literature. Psychiatric Services, 65(1), 24-32.

Lambert, M. J. (1992). Psychotherapy outcome research: implications for integrative and eclectical therapists. In M. R. Goldfried (Ed.), Handbook of psychotherapy integration (pp. 94-129). New York: Basic Books.

Lewinsohn, P. M., Rohde, P., Seeley, J. R., Klein, D. N., \& Gotlib, I. H. (2000). Natural course of adolescent major depressive disorder in a community sample: Predictors of recurrence in young adults. American Journal of Psychiatry, 157(10), 1584-1591.

MacDonell, K. W., \& Prinz, R. J. (2017). A review of technology-based youth and family-focused interventions. Clinical Child and Family Psychology Review, 20(2), 185-200.

Madden, M., Lenhart, A., Duggan, M., Cortesi, S., \& Gasser, U. (2013). Teens and technology 2013 (pp. 1-19). Washington, DC: Pew Internet \& American Life Project.

Melville, K. M., Casey, L. M., \& Kavanagh, D. J. (2010). Dropout from Internet-based treatment for psychological disorders. British Journal of Clinical Psychology, 49(4), 455-471.

Merikangas, K. R., He, J. P., Burstein, M., Swendsen, J., Avenevoli, S., Case, B., et al. (2011). Service utilization for lifetime mental disorders in US adolescents: Results of the National Comorbidity Survey-Adolescent Supplement (NCS-A). Journal of the American Academy of Child \& Adolescent Psychiatry, 50(1), 32-45.
Merikangas, K. R., Nakamura, E. F., \& Kessler, R. C. (2009). Epidemiology of mental disorders in children and adolescents. Dialogues in Clinical Neuroscience, 11(1), 7.

Merry, S. N., Hetrick, S. E., Cox, G. R., Brudevold-Iversen, T., Bir, J. J., \& McDowell, H. (2012a). Cochrane Review: Psychological and educational interventions for preventing depression in children and adolescents. Evidence-Based Child Health: A Cochrane Review Journal, 7(5), 1409-1685.

Newman, M. G., Szkodny, L. E., Llera, S. J., \& Przeworski, A. (2011). A review of technology-assisted self-help and minimal contact therapies for anxiety and depression: is human contact necessary for therapeutic efficacy?. Clinical Psychology Review, 31(1), 89-103.

Ofcom (2013). Children and parents: Media use and attitudes report. London: Office of Communications. 29th November 2017.

Pennant, M. E., Loucas, C. E., Whittington, C., Creswell, C., Fonagy, P., Fuggle, P., et al. (2015). Computerised therapies for anxiety and depression in children and young people: A systematic review and meta analysis. Behaviour Research and Therapy, 67, 1-18.

Perry, Y., Werner-Seidler, A., Calear, A., Mackinnon, A., King, C., Scott, J., et al. (2017). Preventing depression in final year secondary students: School-based randomized controlled trial. Journal of Medical Internet Research, 19(11), e369.

Podina, I. R., Mogoase, C., David, D., Szentagotai, A., \& Dobrean, A. (2016). A meta-analysis on the efficacy of technology mediated CBT for anxious children and adolescents. Journal of RationalEmotive \& Cognitive-Behavior Therapy, 34, 31-50.

Reynolds, S., Wilson, C., Austin, J., \& Hooper, L. (2012). Effects of psychotherapy for anxiety in children and adolescents: A metaanalytic review. Clinical Psychology Review, 32(4), 251-262.

Richards, D., \& Richardson, T. (2012). Computer-based psychological treatments for depression: A systematic review and meta-analysis. Clinical Psychology Review, 32(4), 329e342.

Richardson, T., Stallard, P., \& Velleman, S. (2010). Computerised cognitive behavioural therapy for the prevention and treatment of depression and anxiety in children and adolescents: A systematic review. Clinical Child and Family Psychology Review, 13(3), 275-290.

Rickwood, D., \& Bradford, S. (2012). The role of self-help in the treatment of mild anxiety disorders in young people: An evidencebased review. Psychology Research and Behavior Management, $5,25-36$.

Riper, H., Andersson, G., Christensen, H., Cuijpers, P., Lange, A., \& Eysenbach, G. (2010). Theme issue on e-mental health: A growing field in internet research. Journal of Medical Internet Research, 12(5), e74.

Siddaway, A. P., Wood, A. M., \& Cartwright-Hatton, S. (2014). Involving parents in cognitive-behavioral therapy for child anxiety problems: A case study. Clinical Case Studies, 13(4), 322-335.

Spence, S. H., Donovan, C., \& Brechman-Toussaint, M. (2000). The treatment of childhood social phobia: The effectiveness of a social skills training-based, cognitive-behavioral intervention, with and without parental involvement. Journal of Child Psychology and Psychiatry, 41, 713-726.

Stallard, P. (2005). A Clinician's guide to think good-feel good: Using CBT with children and young people. Chichester: John Wiley \& Sons.

Stallard, P., Porter, J., \& Grist, R. (2018) A smartphone app (BlueIce) for young people (aged 12-17) who self-harm: An Open Phase 1 Trial. JMIR mHealth and uHealth, 6(1), e32.

Thirlwall, K., Cooper, P., Karalus, J., Voysey, M., Willetts, L., \& Creswell, C. (2013). The treatment of child anxiety disorders via guided parent delivered cognitive-behavioural therapy: A randomised controlled trial. British Journal of Psychiatry, 203(6), $436-444$. 
Vigerland, S., Lenhard, F., Bonnert, M., Lalouni, M., Hedman, E., Ahlen, J., et al. (2016a). Internet-delivered cognitive behavior therapy for children and adolescents: A systematic review and meta-analysis. Clinical Psychology Review, 50, 1-10.

Walkup, J. T., Albano, A. M., Piacentini, J., Birmaher, B., Compton, S. N., Sherrill, J. T., et al. (2008). Cognitive behavioral therapy, sertraline, or a combination in childhood anxiety. New England Journal of Medicine, 359(26), 2753-2766.

Watts, S. E., Turnell, A., Kladnitski, N., Newby, J. M., \& Andrews, G. (2015). Treatment-as-usual (TAU) is anything but usual: a metaanalysis of CBT versus TAU for anxiety and depression. Journal of Affective Disorders, 175, 152-167.

Woodward, L. J., \& Fergusson, D. M. (2001). Life course outcomes of young people with anxiety disorders in adolescence. Journal of the American Academy of Child \& Adolescent Psychiatry, 40(9), 1086-1093.

Ye, X. B., Bapuji, S. B., Winters, S. E., Struthers, A., Raynard, M., Metge, C., \& Sutherland, K. (2014). Effectiveness of internetbased interventions for children, youth, and young adults with anxiety and/or depression: A systematic review and meta-analysis. BMC Health Services Research, 14(1), 313.

\section{References of Studies Included in the Meta-analysis}

Bar-Haim, Y., Morag, I., \& Glickman, S. (2011). Training anxious children to disengage attention from threat: A randomized controlled trial. Journal of Child Psychology and Psychiatry, 52(8), 861-869.

Conaughton, R. J., Donovan, C. L., \& March, S. (2017). Efficacy of an internet-based CBT program for children with comorbid High Functioning Autism Spectrum Disorder and anxiety: A randomised controlled trial. Journal of Affective Disorders, 218 , 260-268

De Voogd, E. L., Wiers, R. W., \& Salemink, E. (2017). Online visual search attentional bias modification for adolescents with heightened anxiety and depressive symptoms: A randomized controlled trial. Behaviour Research and Therapy, 92, 57-67.

Fitzgerald, A., Rawdon, C., \& Dooley, B. (2016). A randomized controlled trial of attention bias modification training for socially anxious adolescents. Behaviour Research and Therapy, 84, 1-8.

$\mathrm{Fu}, \mathrm{X} ., \mathrm{Du}, \mathrm{Y} ., \mathrm{Au}, \mathrm{S} .$, \& Lau, J. Y. (2013). Reducing negative interpretations in adolescents with anxiety disorders: A preliminary study investigating the effects of a single session of cognitive bias modification training. Developmental Cognitive Neuroscience, 4 , 29-37.

Hoek, W., Schuurmans, J., Koot, H. M., \& Cuijpers, P. (2012). Effects of Internet-based guided self-help problem-solving therapy for adolescents with depression and anxiety: A randomized controlled trial. PLoS ONE, 7(8), e43485.

Ip, P., Chim, D., Chan, K. L., Li, T. M., Ho, F. K. W., Voorhees, B. W., et al. (2016). Effectiveness of a culturally attuned Internetbased depression prevention program for Chinese adolescents: A randomized controlled trial. Depression and Anxiety, 33(12), $1123-1131$.

LeMoult, J., Colich, N., Joormann, J., Singh, M. K., Eggleston, C., \& Gotlib, I. H. (2017). Interpretation bias training in depressed adolescents: Near-and far-transfer effects. Journal of Abnormal Child Psychology, 46(1), 1-9.

Lenhard, F., Andersson, E., Mataix-Cols, D., Rück, C., Vigerland, S., Högström, J., et al. (2017). Therapist-guided, Internet-delivered cognitive-behavioral therapy for adolescents with obsessivecompulsive disorder: A randomized controlled trial. Journal of the American Academy of Child \& Adolescent Psychiatry, 56(1), $10-19$.
March, S., Spence, S. H., \& Donovan, C. L. (2009). The efficacy of an internet-based cognitive-behavioral therapy intervention for child anxiety disorders. Journal of Pediatric Psychology, 34(5), 474-487.

Merry, S. N., Stasiak, K., Shepherd, M., Frampton, C., Fleming, T., \& Lucassen, M. F. (2012b). The effectiveness of SPARX, a computerised self help intervention for adolescents seeking help for depression: Randomised controlled non-inferiority trial. $B M J$, 344, e2598.

Muris, P., Merckelbach, H., Holdrinet, I., \& Sijsenaar, M. (1998). Treating phobic children: Effects of EMDR versus exposure. Journal of Consulting and Clinical Psychology, 66(1), 193.

Pergamin-Hight, L., Pine, D. S., Fox, N. A., \& Bar-Haim, Y. (2016). Attention bias modification for youth with social anxiety disorder. Journal of Child Psychology and Psychiatry, 57(11), 1317-1325.

Poppelaars, M., Tak, Y. R., Lichtwarck-Aschoff, A., Engels, R. C., Lobel, A., Merry, S. N., et al. (2016). A randomized controlled trial comparing two cognitive-behavioral programs for adolescent girls with subclinical depression: A school-based program (Op Volle Kracht) and a computerized program (SPARX). Behaviour Research and Therapy, 80, 33-42.

Rickhi, B., Kania-Richmond, A., Moritz, S., Cohen, J., Paccagnan, P., Dennis, C., et al. (2015). Evaluation of a spirituality informed e-mental health tool as an intervention for major depressive disorder in adolescents and young adults-A randomized controlled pilot trial. BMC Complementary and Alternative Medicine, 15(1), 450.

Schleider, J., \& Weisz, J. (2017). A single-session growth mindset intervention for adolescent anxiety and depression: 9-month outcomes of a randomized trial. Journal of Child Psychology and Psychiatry, 59(2), 160-170

Scholten, H., Malmberg, M., Lobel, A., Engels, R. C., \& Granic, I. (2016). A randomized controlled trial to test the effectiveness of an immersive $3 \mathrm{D}$ video game for anxiety prevention among adolescents. PLos ONE, 11(1), e0147763.

Schoneveld, E. A., Lichtwarck-Aschoff, A., \& Granic, I. (2017). Preventing childhood anxiety disorders: Is an applied game as effective as a cognitive behavioral therapy-based program?. Prevention Science, 19(2), 1-13.

Schoneveld, E. A., Malmberg, M., Lichtwarck-Aschoff, A., Verheijen, G. P., Engels, R. C., \& Granic, I. (2016). A neurofeedback video game (MindLight) to prevent anxiety in children: A randomized controlled trial. Computers in Human Behavior, 63, 321-333.

Smith, P., Scott, R., Eshkevari, E., Jatta, F., Leigh, E., Harris, V., et al. (2015). Computerised CBT for depressed adolescents: Randomised controlled trial. Behaviour Research and Therapy, 73, 104-110.

Spence, S. H., Donovan, C. L., March, S., Gamble, A., Anderson, R. E., Prosser, S., et al. (2011). A randomized controlled trial of online versus clinic-based CBT for adolescent anxiety. Journal of Consulting and Clinical Psychology, 79(5), 629.

Spence, S. H., Donovan, C. L., March, S., Kenardy, J. A., \& Hearn, C. S. (2017). Generic versus disorder specific cognitive behavior therapy for social anxiety disorder in youth: A randomized controlled trial using internet delivery. Behaviour Research and Therapy, 90, 41-57.

Sportel, B. E., de Hullu, E., de Jong, P. J., \& Nauta, M. H. (2013). Cognitive bias modification versus CBT in reducing adolescent social anxiety: A randomized controlled trial. PLOS ONE, 8(5), e64355.

Stallard, P., Richardson, T., Velleman, S., \& Attwood, M. (2011). Computerized CBT (Think, Feel, Do) for depression and anxiety in children and adolescents: Outcomes and feedback from a pilot randomized controlled trial. Behavioural and Cognitive Psychotherapy, 39(3), 273-284.

Stasiak, K., Hatcher, S., Frampton, C., \& Merry, S. N. (2014). A pilot double blind randomized placebo controlled trial of a prototype 
computer-based cognitive behavioural therapy program for adolescents with symptoms of depression. Behavioural and Cognitive Psychotherapy, 42(4), 385-401.

Tillfors, M., Andersson, G., Ekselius, L., Furmark, T., Lewenhaupt, S., Karlsson, A., et al. (2011). A randomized trial of internet-delivered treatment for social anxiety disorder in high school students. Cognitive Behaviour Therapy, 40(2), 147-157.

Vigerland, S., Ljótsson, B., Thulin, U., Öst, L. G., Andersson, G., \& Serlachius, E. (2016b). Internet-delivered cognitive behavioural therapy for children with anxiety disorders: A randomised controlled trial. Behaviour Research and Therapy, 76, 47-56.

Wannachaiyakul, S., Thapinta, D., Sethabouppha, H., Thungjaroenkul, P., \& Likhitsathian, S. (2017). Randomized controlled trial of computerized cognitive behavioral therapy program for adolescent offenders with depression. Pacific Rim International Journal of Nursing Research, 21(1), 32-43.

Waters, A., Zimmer-Gembeck, M., Craske, M., Pine, D., Bradley, B. P., \& Mogg, K. (2016). A preliminary evaluation of a home-based, computer-delivered attention training treatment for anxious children living in regional communities. Journal of Experimental Psychopathology, 7(3), 511-527.

Waters, A. M., Pittaway, M., Mogg, K., Bradley, B. P., \& Pine, D. S. (2013). Attention training towards positive stimuli in clinically anxious children. Developmental Cognitive Neuroscience, 4, 77-84.

Waters, A. M., Zimmer-Gembeck, M. J., Craske, M. G., Pine, D. S., Bradley, B. P., \& Mogg, K. (2015). Look for good and never give up: A novel attention training treatment for childhood anxiety disorders. Behaviour Research and Therapy, 73, 111-123.

Wright, B., Tindall, L., Littlewood, E., Allgar, V., Abeles, P., Trépel, D., \& Ali, S. (2017). Computerised cognitive-behavioural therapy for depression in adolescents: Feasibility results and 4-month outcomes of a UK randomised controlled trial. BMJ Open, 7(1), e012834.

Wuthrich, V. M., Rapee, R. M., Cunningham, M. J., Lyneham, H. J., Hudson, J. L., \& Schniering, C. A. (2012). A randomized controlled trial of the Cool Teens CD-ROM computerized program for adolescent anxiety. Journal of the American Academy of Child \& Adolescent Psychiatry, 51(3), 261-270.

Yang, W., Zhang, J. X., Ding, Z., \& Xiao, L. (2016). Attention bias modification treatment for adolescents with major depression: A randomized controlled trial. Journal of the American Academy of Child \& Adolescent Psychiatry, 55(3), 208-218. 